\title{
The Boomerang Attacks on BLAKE and BLAKE2
}

\author{
Yonglin Hao \\ Department of Computer Science and Technology, Tsinghua Universtiy, Beijing 100084, China \\ haoyl12@mails.tsinghua.edu.cn
}

\begin{abstract}
In this paper, we study the security margins of hash functions BLAKE and BLAKE2 against the boomerang attack. We launch boomerang attacks on all four members of BLAKE and BLAKE2, and compare their complexities. We propose 8.5-round boomerang attacks on both BLAKE-512 and BLAKE2b with complexities $2^{464}$ and $2^{474}$ respectively. We also propose 8 -round attacks on BLAKE256 with complexity $2^{198}$ and 7.5 -round attacks on BLAKE2s with complexity $2^{184}$. We verify the correctness of our analysis by giving practical 6.5-round Type I boomerang quartets for each member of BLAKE and BLAKE2.

According to our analysis, some tweaks introduced by BLAKE2 have increased its resistance against boomerang attacks to a certain extent. But on the whole, BLAKE still has higher a secure margin than BLAKE2.
\end{abstract}

\section{Introduction}

Cryptographic hash functions (simply referred as hash functions) are playing a significant role in the modern cryptology. They are indispensable in achieving secure systems such as digital signatures, message authentication codes and so on. In the cryptanalysis of hash functions, one of the greatest breakthrough was made by Wang et al. in 2005 when they successfully launched collision attacks on widely used hash functions MD5 [1] and SHA-1 2]. After that, the analytic methods against hash functions have been greatly improved which threatens the security of existing hash functions. To cope with this situation, NIST proposed the transition from SHA-1 to SHA-2. Furthermore, NIST also launched the SHA-3 competition to develop a new hash standard. After years' analysis, five proposals entered the final round of SHA-3 and the one named Keccak became the new SHA-3 standard in 2012 [3].

The BLAKE hash function [4] was one of the five finalists of the SHA-3 competition [5. Although it was not selected as the SHA-3 standard, along with the other finalists, BLAKE is assumed to be a very strong hash function with high security margin and very good performance in software.

BLAKE2 [6] is a new family of hash functions based on BLAKE. According to [6], the main objective of BLAKE2 is to provide a number of parameters for use in applications without the need of additional constructions and modes, and also to speed-up even further the hash function to a level of compression rate close to MD5.

Ever since its proposal, BLAKE has attracted a considerable amount of cryptanalysis, such as impossible differential attack [7, differential attack [8], collision, preimage [9] etc. There is also a boomerang distinguisher on BLAKE-32 given by Biryukov et al. in [10] but some incompatible problems were pointed out by Leurent in [11. Despite of the incompatibilities, [10] indicates that the boomerang method may have good efficiency in analyzing the BLAKE family. Recently, Bai et al. have given the first valid 7-round and 8-round boomerangs for BLAKE-256 [12].

As to BLAKE2, Guo et al. [13] have given a thorough security analysis of it. In their paper, they applied almost all the existing attacks on BLAKE to BLAKE2. According to their results, the tweaks introduced by BLAKE2, if analyzed separately, reduce the security of the version in some theoretical attacks. Some cryptanalysis methods manage to reach more rounds for BLAKE2 than BLAKE. BLAKE seems to have better resistance than BLAKE2 against various cryptanalysis methods. However, 13] did not evaluate the security margin of the two hash function families under the boomerang method and this is what we are going to do in this paper.

The original boomerang attack was introduced by Wagner in 1999 [14 as a tool for the cryptanalysis of block ciphers. It is an adaptive chosen plaintext and ciphertext attack utilizing differential cryptanalysis. 
Later, Kelsey et al. 15 developed the original version into a chosen plaintext attack called the amplified boomerang attack. Developments were also made by Biham et al. in [16] and [17].

During the past few years, the idea of the boomerang attack has been applied to many hash functions. Biryukov et al. [10] and Lamberger et al. 18] independently applied the boomerang attack to BLAKE-32 and SHA-256. The SHA-256 result was later improved by Biryukov et al. in [19]. Ever after, we saw the boomerang results on many hash functions such as SIME-512 [20, HAVAL [21, RIPEMD-128/160 [22], HAS-160 21], Skein-256/512 2324], SM3 25|26] and BLAKE-256 [12]. The boomerang attack has become a common tool for analyzing various hash functions.

Our contribution. We reevaluate the boomerang attack on BLAKE-256 in [12] and apply the method to the keyed permutations of all BLAKE and BLAKE2 members namely BLAKE-256, BLAKE-512, BLAKE2s and BLAKE2b. We construct boomerang distinguishers for 8.5-round keyed permutation of BLAKE-512 and BLAKE2b (both from round 2.5 to 11). The complexity for attacking BLAKE-512 is $2^{464}$ and that for BLAKE2b is $2^{474}$. We also present 7.5-round attack on BLAKE2s (round 2.5 to 10 ) with complexity $2^{184}$. Besides, we lower the complexity of the 8-round BLAKE-256 result in [12] from $2^{200}$ to $2^{198}$ with slight modification of the differential characteristic. We present our boomerang results along with previous ones in Table 1 As can be seen, some tweaks introduced by BLAKE2 have surprisingly increased its resistance against boomerang attacks to a certain extent. But, since BLAKE has more rounds, the secure margin of BLAKE is still higher than that of BLAKE2.

Table 1. All existing boomerang results on BLAKE and BLAKE2.

\begin{tabular}{|c|c|c|c|c|}
\hline Hash function & Target & Rounds & Time & Source \\
\hline \multirow{9}{*}{ BLAKE-256 } & $\mathrm{CF}$ & 6 & $2^{102}$ & \multirow{6}{*}{10} \\
\hline & $\mathrm{CF}$ & $6.5^{*}$ & $2^{184}$ & \\
\hline & $\mathrm{CF}$ & $7^{*}$ & $2^{232}$ & \\
\hline & KP & 6 & $2^{11.75}$ & \\
\hline & $\mathrm{KP}$ & $7^{*}$ & $2^{122}$ & \\
\hline & KP & $8^{*}$ & $2^{242}$ & \\
\hline & $\mathrm{KP}$ & 7 & $2^{37 * *}$ & \multirow[b]{2}{*}{12} \\
\hline & $\mathrm{KP}$ & 8 & $2^{200}$ & \\
\hline & KP & 8 & $2^{198}$ & This paper \\
\hline BLAKE2s & KP & 7.5 & $2^{184}$ & This paper \\
\hline BLAKE-512 & $\mathbf{K P}$ & 8.5 & $2^{464}$ & This paper \\
\hline BLAKE2b & $\mathbf{K P}$ & 8.5 & $2^{474}$ & This paper \\
\hline \multicolumn{5}{|c|}{ KP: Keyed Permutation } \\
\hline \multicolumn{5}{|c|}{ CF: Compression Function } \\
\hline \multicolumn{5}{|c|}{$\begin{array}{l}\text { *: there are some incompatible problems in their attacks } \\
\text { **: this is the complexity for the Type III boomerang } \\
\text { while others are of Type I. }\end{array}$} \\
\hline
\end{tabular}

Organization of the Paper. In Section2, we briefly introduce the round functions of BLAKE and BLAKE2, and provide the overview of the boomerang attack. Section 3 describes the way that we deduce the differential characteristics and the process of building the boomerang distinguishers. Finally, we conclude our paper in Section 4

\section{Preliminary}

In the first part of this section, we make a brief introduction of the two families of hash functions, BLAKE and BLAKE2. Since our boomerang analysis mainly focus on the keyed permutation of BLAKE and BLAKE2, which excludes the Initialization and Finalization procedures, we only introduce the round functions in this section. We refer the readers to [4] and [6] for information about initialization and finalization phases. We also give some notations that are used through this paper. 
In the second part of this section, we review the procedure of the boomerang attack on hash functions and give some definitions that we use in the description of our attacks.

\subsection{The Round Functions of BLAKE and BLAKE2}

BLAKE and BLAKE2 share many similarities. As the successor of BLAKE, BLAKE2 has a 32-bit version (BLAKE2s) and a 64-bit version (BLAKE2b), corresponding to BLAKE-256 and BLAKE-512 of BLAKE respectively. Both BLAKE and BLAKE2 process 16-word message blocks. However, differences can be witnessed at every level including internal permutation, compression function, and hash function construction. Some notations have to be introduced first:

$\leftarrow$ variable assignment;

+ modular $2^{32}$ or $2^{64}$ addition (according to the word length);

- modular $2^{32}$ or $2^{64}$ subtraction (according to the word length);

$\oplus$ bitwise exclusive or;

$\lll n$ cyclic shift $n$ bits towards the most significant bit;

$\ggg n$ cyclic shift $n$ bits towards the least significant bit;

$\wedge$ bitwise AND operation for words.

The Round functions of both BLAKE and BLAKE2 process a state of 16 64-bit or 32-bit words represented by a $4 \times 4$ matrix as follows:

$$
V=\left(\begin{array}{cccc}
v_{0} & v_{1} & v_{2} & v_{3} \\
v_{4} & v_{5} & v_{6} & v_{7} \\
v_{8} & v_{9} & v_{10} & v_{11} \\
v_{12} & v_{13} & v_{14} & v_{15}
\end{array}\right) .
$$

In the remainder of this paper, we denote the 16-word intermediate state by the capital letters such as $V, T V$ and $M$ for message block. Single 64-bit or 32-bit words are denoted by small letters such as $v, t v$ and $m$ for message words. We also refer to the $i$-th bit of a word $v(i=0, \cdots 31$ or 63 from the least significant to the most significant) as $v[i]$.

Once the state $V$ is initialized, $V$ is processed by several rounds $(10,12,14,16$ for BLAKE2s, BLAKE2b, BLAKE-256, BLAKE512 respectively) of $G$ functions, which means computing

$$
\begin{aligned}
& G_{0}\left(v_{0}, v_{4}, v_{8}, v_{12}\right), G_{1}\left(v_{1}, v_{5}, v_{9}, v_{13}\right), G_{2}\left(v_{2}, v_{6}, v_{10}, v_{14}\right), G_{3}\left(v_{3}, v_{7}, v_{11}, v_{15}\right) \\
& G_{4}\left(v_{0}, v_{5}, v_{10}, v_{15}\right), G_{5}\left(v_{1}, v_{6}, v_{11}, v_{12}\right), G_{6}\left(v_{2}, v_{7}, v_{8}, v_{13}\right), G_{7}\left(v_{3}, v_{4}, v_{9}, v_{14}\right)
\end{aligned}
$$

where $G_{i}(a, b, c, d), i=0, \cdots, 7$ differ among BLAKE2s, BLAKE2b, BLAKE-256, BLAKE512 and are all listed in Table 2. The $\sigma_{r}$ in Step 1 and 5 of the $G_{i}$ function in Table 2 belongs to the set of permutations as defined in Table 3 . At round $r>9$, the permutation used is $\sigma_{r} \bmod 10$ (for example, if $r=11$, the permutation

\begin{tabular}{|c|c|c|c|c|}
\hline Step & BLAKE-256 & BLAKE2s & BLAKE-512 & BLAKE2b \\
\hline 1 & $a=a+b+\left(m_{\sigma_{r}(2 i)} \oplus c_{\sigma_{r}(2 i+1)}\right)$ & $a=a+b+m_{\sigma_{r}(2 i)}$ & $a=a+b+\left(m_{\sigma_{r}(2 i)} \oplus c_{\sigma_{r}(2 i+1)}\right)$ & $a=a+b+m_{\sigma_{r}(2 i)}$ \\
\hline 2 & $d=(d \oplus a) \ggg 16$ & $d=(d \oplus a) \ggg 16$ & $d=(d \oplus a) \ggg 32$ & $d=(d \oplus a) \ggg 32$ \\
\hline 3 & $c=c+d$ & $c=c+d$ & $c=c+d$ & $c=c+d$ \\
\hline 4 & $b=(b \oplus c) \ggg 12$ & $b=(b \oplus c) \ggg 12$ & $b=(b \oplus c) \ggg 25$ & $b=(b \oplus c) \ggg 24$ \\
\hline 5 & $a=a+b+\left(m_{\sigma_{r}(2 i+1)} \oplus c_{\sigma_{r}(2 i)}\right)$ & $a=a+b+m_{\sigma_{r}(2 i+1)}$ & $a=a+b+\left(m_{\sigma_{r}(2 i+1)} \oplus c_{\sigma_{r}(2 i)}\right)$ & $a=a+b+m_{\sigma_{r}(2 i+1)}$ \\
\hline 6 & $d=(d \oplus a) \ggg 8$ & $d=(d \oplus a) \ggg 8$ & $d=(d \oplus a) \ggg 16$ & $d=(d \oplus a) \ggg 16$ \\
\hline 7 & $c=c+d$ & $c=c+d$ & $c=c+d$ & $c=c+d$ \\
\hline 8 & $b=(b \oplus c) \ggg 7$ & $b=(b \oplus c) \ggg 7$ & $b=(b \oplus c) \ggg 11$ & $b=(b \oplus c) \ggg 63$ \\
\hline
\end{tabular}
$\sigma_{11} \bmod 10=\sigma_{1}$ is used).

Table 2. The $G_{i}$ Functions of BLAKE-256, BLAKE2s, BLAKE-512, BLAKE2b

Since we need detailed analysis of the intermediate states, we further breakdown the round functions. We denote the state after $r$ rounds of iterations by $V^{r}(r=0,1, \cdots)$. Then, $T V^{r}$ is acquired after the first 4 
steps of $G_{0, \cdots, 3}$ and $V^{r+0.5}$ is computed after $G_{0, \cdots, 3}$ are completed. Similarly, we can compute $T V^{r+0.5}$ from $V^{r+0.5}$ by applying steps $1,2,3,4$ of $G_{4, \cdots, 7}$ and further compute $V^{r+1}$ by finishing $G_{4, \cdots, 7}$. This representation is illustrated as (1) and (2).

$$
\begin{aligned}
& G_{0, \cdots, 3}: V^{r} \stackrel{\text { Steps } 1, \cdots, 4}{\longrightarrow} T V^{r} \stackrel{\text { Steps } 5, \cdots, 8}{\longrightarrow} V^{r+0.5} \\
& G_{4, \cdots, 7}: V^{r+0.5} \stackrel{\text { Steps } 1, \cdots, 4}{\longrightarrow} T V^{r+0.5} \stackrel{\text { Steps } 5, \cdots, 8}{\longrightarrow} V^{r+1}
\end{aligned}
$$

In this way, we can refer to any intermediate state word of any round easily.

Table 3. The definition of $\sigma_{r}$ where $r=0, \cdots, 9$.

\begin{tabular}{c|cccccccccccccccc}
\hline$\sigma_{0}$ & 0 & 1 & 2 & 3 & 4 & 5 & 6 & 7 & 8 & 9 & 10 & 11 & 12 & 13 & 14 & 15 \\
$\sigma_{1}$ & 14 & 10 & 4 & 8 & 9 & 15 & 13 & 6 & 1 & 12 & 0 & 2 & 11 & 7 & 5 & 3 \\
$\sigma_{2}$ & 11 & 8 & 12 & 0 & 5 & 2 & 15 & 13 & 10 & 14 & 3 & 6 & 7 & 1 & 9 & 4 \\
$\sigma_{3}$ & 7 & 9 & 3 & 1 & 13 & 12 & 11 & 14 & 2 & 6 & 5 & 10 & 4 & 0 & 15 & 8 \\
$\sigma_{4}$ & 9 & 0 & 5 & 7 & 2 & 4 & 10 & 15 & 14 & 1 & 11 & 12 & 6 & 8 & 3 & 13 \\
$\sigma_{5}$ & 2 & 12 & 6 & 10 & 0 & 11 & 8 & 3 & 4 & 13 & 7 & 5 & 15 & 14 & 1 & 9 \\
$\sigma_{6}$ & 12 & 5 & 1 & 15 & 14 & 13 & 4 & 10 & 0 & 7 & 6 & 3 & 9 & 2 & 8 & 11 \\
$\sigma_{7}$ & 13 & 11 & 7 & 14 & 12 & 1 & 3 & 9 & 5 & 0 & 15 & 4 & 8 & 6 & 2 & 10 \\
$\sigma_{8}$ & 6 & 15 & 14 & 9 & 11 & 3 & 0 & 8 & 12 & 2 & 13 & 7 & 1 & 4 & 10 & 5 \\
$\sigma_{9}$ & 10 & 2 & 8 & 4 & 7 & 6 & 1 & 5 & 15 & 11 & 9 & 14 & 3 & 12 & 13 & 0
\end{tabular}

\subsection{The Boomerang Attack}

About the boomerang attack on hash functions, we mainly review the known-related-key boomerang method given in [19. We consider the compression function, denoted by $C F$, as $C F(M, K)=E(M, K)+M$ and that it can be decomposed into two sub-functions as $C F=C F_{1} \circ C F_{0}$. In this way, we can start from the middle steps since $M$ and the key $K$ can be chosen randomly [1923. Then we have a backward (top) differential characteristic $\left(\beta, \beta_{k}\right) \rightarrow \alpha$ with probability $p$ for $C F_{0}^{-1}$, and a forward (bottom) differential characteristic $\left(\gamma, \gamma_{k}\right) \rightarrow \delta$ with probability $q$ for $C F_{1}$. Finally, we can launch the known-related-key boomerang attack with these two differential characteristics as follows:

1. Choose randomly a intermediate state $\left(X_{1}, K_{1}\right)$ and compute $\left(X_{i}, K_{i}\right), i=2,3,4$ by $X_{3}=X_{1} \oplus \beta$, $X_{2}=X_{1} \oplus \gamma, X_{4}=X_{3} \oplus \gamma$, and $K_{3}=K_{1} \oplus \beta_{k}, K_{2}=K_{1} \oplus \gamma_{k}, K_{4}=K_{3} \oplus \gamma_{k}$.

2. Compute backward from $\left(X_{i}, K_{i}\right)$ and obtain $P_{i}$ by $P_{i}=C F_{0}^{-1}\left(X_{i}, K_{i}\right)(i=1,2,3,4)$.

3. Compute forward from $\left(X_{i}, K_{i}\right)$ and obtain $C_{i}$ by $C_{i}=C F_{1}\left(X_{i}, K_{i}\right)(i=1,2,3,4)$.

4. Check whether $P_{1} \oplus P_{3}=P_{2} \oplus P_{4}=\alpha$ and $C_{1} \oplus C_{2}=C_{3} \oplus C_{4}=\delta$.

It can be deduced that $P_{1} \oplus P_{3}=P_{2} \oplus P_{4}=\alpha$ and $C_{1} \oplus C_{2}=C_{3} \oplus C_{4}=\delta$ hold with probability at least $p^{2}$ in the backward direction and $q^{2}$ in the forward direction. Therefore, the attack succeeds with probability $p^{2} q^{2}$ when assuming that the differential characteristics are independent.

According $\mathrm{H}$. Yu et al. in [24], for a $n$-bit random permutation, there are three types of boomerang distinguishers:

- Type I: A quartet satifies $P_{1} \oplus P_{3}=P_{2} \oplus P_{4}=\alpha$ and $C_{1} \oplus C_{2}=C_{3} \oplus C_{4}=\delta$ for fixed differences $\alpha$ and $\delta$. In this case, the generic complexity is $2^{n}$.

- Type II: Only $C_{1} \oplus C_{2}=C_{3} \oplus C_{4}$ is satisfied (This property is also called zero-sum or second-order differential collision). In this case, the complexity for obtaining such a quartet is $2^{n / 3} 27$.

- Type III: A quartet satisfied $P_{1} \oplus P_{3}=P_{2} \oplus P_{4}$ and $C_{1} \oplus C_{2}=C_{3} \oplus C_{4}$. In this case, the best known still takes time $2^{n / 2}$.

We only study the Type I boomerang distinguisher in this paper. Besides, the complexity $2^{37}$ of the 7 -round boomerang in 12 is actually the complexity for a Type III boomerang attack. The Type I complexity for the 7 -round attack should be $2^{2 \times(1+4+16+1)}=2^{44}$ according to their methods. 


\section{The Boomerang Attacks on BLAKE and BLAKE2}

In this section, we describe our boomerang attacks on BLAKE and BLAKE2. We only illustrate our strategies by comparing BLAKE-512 and BLAKE2b while those of BLAKE-256 and BLAKE2s can be deduced accordingly. Details are presented in Appendix A

\subsection{Construction of Differential Characteristics}

The very first step for the boomerang attack is constructing two differential characteristics with high probability. Since BLAKE and BLAKE2 are ARX hash functions (only use three simple operations namely Modular Add "+", Rotation "ゆ" and XOR " $\oplus$ "), we can use the XOR difference and deduce the difference linearly by considering the only nonlinear operation "+" as similar linear operation " $\oplus$ ".

The XOR difference in this paper is represented in two forms as follow:

- Hex form: such as $\Delta v=0 \mathrm{x} 8003$ indicates that bits $v[0,1,15]$ of the word $v$ are active (having non-zero XOR difference).

- Numeric form: such as $\Delta v=(15,1,0)$ is equivalent to $\Delta v=0 \mathrm{x} 8003$ in hex form. Besides, if $\Delta v=0 \mathrm{x} 0$ in hex form, we denoted by $\Delta v=\phi$ in numeric form.

The numeric form is mainly used to describe the differential characteristics since it has better outlook and can save some space. But in practice, we use the hex form to linearly deduce differential characteristics. For example, in the $G$ function of BLAKE-512, we have

$$
t a=a+b+\left(m_{i} \oplus c_{j}\right)
$$

where $c_{j}$ is constant. Suppose we have acquired the differences $\Delta a, \Delta b$ and $\Delta m_{i}$, we can dedcue $\Delta t a$ as

$$
\Delta t a=\Delta a \oplus \Delta b \oplus \Delta m_{i} .
$$

Once we have determined the difference of the message block $\Delta M$ and that of a intermediate state $\Delta V^{r}$ $(r=0,0.5,1, \cdots)$, we can linearly extend the difference backward and forward.

We construct the two differential characteristics for the boomerang attack, where the top differential characteristic is from round 2.5 to 6.5 and bottom differential difference is from 6.5 to 11 . We denote the difference of the top by $\Delta^{t} V^{r}(r \in[2.5,6.5])$ and that of the bottom by $\Delta^{b} V^{r}(r \in[6.5,11])$. Similarly, the difference for the message block is denoted as $\Delta^{t} M$ in the top characteristic and $\Delta^{b} M$ in the bottom characteristic. The main procedures for our characteristic construction can be summarized as follows:

Import Difference: We first import simple difference to message block $\Delta^{b} M\left(\Delta^{t} M\right)$ and the intermediate state $\Delta^{b} V^{8}\left(\Delta^{t} V^{4}\right)$.

Linear Extension: After we have determined $\Delta^{b} M\left(\Delta^{t} M\right)$ and $\Delta^{b} V^{8}\left(\Delta^{t} V^{4}\right)$, we extend the difference backward to round $6.5(2.5)$ and forward to round 11 (6.5) to acquire the whole bottom (top) differential characteristic.

Construct the Bottom Differential Characteristic: In order to lower the complexity, we only import 1-bit differences to both $\Delta^{b} M$ and $\Delta^{b} V^{8}$. The selection of active bits is based on Observation 1 in [10].

We found that $m_{11}$ of the 16 message words, namely $m_{0}, \cdots, m_{15}$, appears at Step 1 in $G_{2}$ at round 8 and also appears at Step 5 in $G_{4}$ at round 9. So, the first step of our construction is importing 1-bit difference to $m_{11}$ and $v_{2}^{8}$ as

$$
\Delta^{b} m_{11}=\Delta^{b} v_{2}^{8}=(63) .
$$

In this way, according to Observation 1 in [10, we can pass round 8 and 9 with probability $2^{-1}$. Then, we set $\Delta^{b} m_{i}=\phi(i \in\{0,1, \cdots, 15\} \backslash\{11\})$ and $\Delta^{b} v_{j}^{8}=\phi(j \in\{0,1, \cdots, 15\} \backslash\{2\})$. Now that $\Delta^{b} M$ and $\Delta^{b} V^{8}$ are settled, we can linearly extend the difference backward to $\Delta^{b} V^{6.5}$ and forward to $\Delta^{b} V^{11}$. This method can be applied to both BLAKE-512 and BLAKE2b. We present the bottom characteristics of BLAKE-512 and BLAKE2b as Table 4 and 5 in Appendix A respectively. 
For BLAKE-256 and BLAKE2s, we can also import difference to $\Delta^{b} M$ and $\Delta^{b} V^{8}$ as

$$
\Delta^{b} m_{11}=\Delta^{b} v_{2}^{8}=(31) .
$$

and linearly deduce the whole bottom differential characteristics. The differential characteristic for BLAKE256 mounts to round 10.5 and BLAKE2s reaches round 10 since it only has 10 rounds in total according to 6. Refer to Table 6 and Table 7 in Appendix A for detailed descriptions.

Construct the Top Differential Characteristic: The top differential characteristic starts from $\Delta^{t} V^{2.5}$ and ends at $\Delta^{t} V^{6.5}$. The strategy of constructing the top differential characteristic is similar to that of its bottom counterpart. We found that $m_{5}$ appears at Step 1 in $G_{1}$ at round 4 and also appears at Step 5 in $G_{5}$ at round 5 , so we decide to import the 1-bit difference at $m_{5}$ and $v_{1}^{4}$. We assign that

$$
\Delta^{t} m_{5}=\Delta^{t} v_{1}^{4}=(y) \text {, where } y \in\{0, \cdots, 63\} .
$$

and that $\Delta^{b} m_{i}=\phi(i \in\{0, \cdots, 15\} \backslash\{5\})$ and $\Delta^{b} v_{j}^{4}=\phi(j \in\{0, \cdots, 15\} \backslash\{1\})$. Then, we can linearly extend the difference backward and forward. The position of the active bit $y$ in (5) requires careful selection. In order to avoid incompatible problems and enhance the efficiency of the attack, $y$ must meet the following conditions:

1. When linearly extend the difference from $\Delta^{t} V^{4}(y)$ to $\Delta^{t} V^{6.5}(y)$, make sure that

$$
\Delta^{b} v_{i}^{6.5} \wedge \Delta^{t} v_{i}^{6.5}(y)=0 \mathrm{x} 0 \text {, for all } i \in\{0, \cdots, 15\} .
$$

This restriction avoid the contradictions in the intersection part of the two differential characteristics.

2. (Only for BLAKE-512) Make sure that the constants $c_{10}$ and $c_{7}$ satisfies:

$$
c_{10}[y]=\neg c_{7}[y] .
$$

According to the linear extension, we have $\Delta^{t} v_{1}^{3.5}=\phi$. It requires $\left(m_{5} \oplus c_{10}\right)[y]=\neg\left(m_{5} \oplus c_{7}\right)[y]$, so (7) must be satisfied.

3. (Only for BLAKE2b) When linearly extend to $\Delta^{t} V^{3.5}, \Delta^{t} v_{1}^{3.5}$ should be set to

$$
\Delta^{t} v_{1}^{3.5}=\Delta^{t} m_{5}+\Delta^{t} m_{5}
$$

instead of 0x0. Because BLAKE2b omit the use of constant, the difference can not be eliminated at $v_{1}^{3.5}$.

The available $y$ s satisfying conditions 1 and 2 compose a set $\mathbb{X}_{512}$, and those satisfying conditions 1 and 3 compose a set $\mathbb{X}_{2 b}$. According to our analysis, $\mathbb{X}_{512}$ has 13 elements and $\mathbb{X}_{2 b}$ has 40 elements. We present $\mathbb{X}_{512}$ and $\mathbb{X}_{2 b}$ along with the corresponding top differential characteristics in Table 8 and Table 9 in Appendix A

Using the same method, we can also acquire the available $y$ s for BLAKE-256 $\left(\mathbb{X}_{256}\right)$ and BLAKE2s $\left(\mathbb{X}_{2 s}\right)$. We illustrate $\mathbb{X}_{256}$ and $\mathbb{X}_{2 s}$ along with their characteristics in Table 10 and Table 11 in Appendix A.

\subsection{Finding the Boomerang Quartet Using Message Modification Technique}

The goal of our boomerang attack is to find a quartet, denoted by $\left({ }_{a} V^{2.5}{ }_{b} V^{2.5},{ }_{c} V^{2.5},{ }_{d} V^{2.5}\right)$, and the message blocks $\left({ }_{a} M,{ }_{b} M,{ }_{c} M,{ }_{d} M\right)$ that satisfies

$$
\begin{aligned}
{ }_{a} V^{2.5} \oplus{ }_{c} V^{2.5} & ={ }_{b} V^{2.5} \oplus{ }_{d} V^{2.5}=\Delta^{t} V^{2.5} \\
{ }_{a} M \oplus{ }_{c} M & ={ }_{b} M \oplus{ }_{d} M=\Delta^{t} M \\
{ }_{a} M \oplus{ }_{b} M & ={ }_{c} M \oplus{ }_{d} M=\Delta^{b} M
\end{aligned}
$$

and, after 8.5 rounds, the corresponding quartet $\left({ }_{a} V^{11},{ }_{b} V^{11},{ }_{c} V^{11},{ }_{d} V^{11}\right)$ satisfies

$$
{ }_{a} V^{11} \oplus{ }_{b} V^{11}={ }_{c} V^{11} \oplus{ }_{d} V^{11}=\Delta^{b} V^{11} .
$$


We start by searching for appropriate ${ }_{a} V^{6.5}$ and ${ }_{a} M$. Once ${ }_{a} V^{6.5}$ is determined, ${ }_{b} V^{6.5},{ }_{c} V^{6.5}$ and ${ }_{d} V^{6.5}$ can be settled directly since

$$
\begin{aligned}
& { }_{a} V^{6.5} \oplus_{c} V^{6.5}={ }_{b} V^{6.5} \oplus_{d} V^{6.5}=\Delta^{t} V^{6.5} \\
& { }_{a} V^{6.5} \oplus_{b} V^{6.5}={ }_{c} V^{6.5} \oplus{ }_{d} V^{6.5}=\Delta^{b} V^{6.5}
\end{aligned}
$$

Once ${ }_{a} M$ is determined, ${ }_{b} M,{ }_{c} M$ and ${ }_{d} M$ can also be determined according to $(9)$ and $(10)$. The step of finding the quartet is as follows:

1. Construct an intermediate state, denoted by $V^{6.5}$, and a message block, denoted by $M$, by setting the values of their 16 words randomly.

2. Compute backward to $T V^{6}$ and $V^{6}$, and forward to $T V^{6.5}, V^{7}$. During the process, if one of bit conditions, which are deduced from the top and bottom characteristics, is violated, we can fix it by modifying the words of $V^{6.5}$ or $M$. This process is called the "message modification".

3. After all conditions between round 6 and 7 are satisfied, we assign that ${ }_{a} V^{6.5} \leftarrow V^{6.5}$ and ${ }_{a} M \leftarrow M$. We also assign corresponding values to ${ }_{b} V^{6.5},{ }_{c} V^{6.5},{ }_{d} V^{6.5}$ according to (11) (12) and to ${ }_{b} M,{ }_{c} M,{ }_{d} M$ according to (9) (10).

4. Having acquired $\left({ }_{a} V^{6.5},{ }_{b} V^{6.5},{ }_{c} V^{6.5}{ }_{d} V^{6.5}\right)$ and $\left({ }_{a} M,{ }_{b} M,{ }_{c} M,{ }_{d} M\right)$, we compute backward to round 2.5 . During the process, we check whether the differences of the intermediate states conform to the top differential characteristic. Once a contradiction is detected, go back to 1 .

5. Compute forward from round 6.5 to round 11. During the computation, we check whether differences of the intermediate states conform to the bottom differential characteristic. Once a contradiction is detected, go back to 1 . Otherwise, output the quartet $\left({ }_{a} V^{11},{ }_{b} V^{11},{ }_{c} V^{11},{ }_{d} V^{11}\right)$.

Complexity analysis. For all 4 members of BLAKE and BLAKE2, there are 30 conditions in $\Delta^{b} V^{6} \rightarrow$ $\Delta^{t} V^{6.5}$. 29 of them can be fixed using the message modification technique. All two conditions in $\Delta^{t} V^{6} \rightarrow$ $\Delta^{t} V^{5.5}$ can be fixed as well. Similarly, all 40 conditions in $\Delta^{b} V^{6.5} \rightarrow \Delta^{b} V^{7}$ and 2 out of 6 conditions in $\Delta^{b} V^{7} \rightarrow \Delta^{b} V^{7.5}$ can be fixed. Then, we analyze the four members separately as follows:

BLAKE-512: In the bottom characteristic, there are 4 unfixed conditions in $\Delta^{b} V^{7} \rightarrow \Delta^{b} V^{7.5}, 1$ in $\Delta^{b} V^{9.5} \rightarrow$ $\Delta^{b} V^{10}, 24$ in $\Delta^{b} V^{10} \rightarrow \Delta^{b} V^{10.5}$ and 138 in $\Delta^{b} V^{10.5} \rightarrow \Delta^{b} V^{11}$, which is 167 in total. In the top characteristics, the situation is as follows: 1 unfixed condition in $\Delta^{t} V^{4.5} \rightarrow \Delta^{t} V^{4}, 2$ in $\Delta^{t} V^{4} \rightarrow \Delta^{t} V^{3.5}, 11$ in $\Delta^{t} V^{3.5} \rightarrow \Delta^{t} V^{3}$ and 51 in $\Delta^{t} V^{3} \rightarrow \Delta^{t} V^{2.5}$, which is 65 in total. So, the complexity of the boomerang attack on BLAKE-512 is $2^{(167+65) \times 2}=2^{464}$.

BLAKE2b: In the bottom characteristic, there are 4 unfixed conditions in $\Delta^{b} V^{7} \rightarrow \Delta^{b} V^{7.5}, 1$ in $\Delta^{b} V^{9.5} \rightarrow$ $\Delta^{b} V^{10}, 24$ in $\Delta^{b} V^{10} \rightarrow \Delta^{b} V^{10.5}$ and 124 in $\Delta^{b} V^{10.5} \rightarrow \Delta^{b} V^{11}$, which is 153 in total. The top differential characteristic is slightly different from BLAKE-512 after finishing the procedure $\Delta^{t} V^{6.5} \rightarrow \Delta^{t} V^{4}$. There are 3 unfixed conditions in $\Delta^{t} V^{4} \rightarrow \Delta^{t} V^{3.5}, 13$ in $\Delta^{t} V^{3.5} \rightarrow \Delta^{t} V^{3}$ and 67 in $\Delta^{t} V^{3} \rightarrow \Delta^{t} V^{2.5}$. So the number of unfixed conditions in the top characteristic enhances to $1+3+13+67=84$. The complexity of the boomerang attack on BLAKE2b is $2^{(153+84) \times 2}=2^{474}$.

BLAKE-256: Similar to BLAKE-512, the bottom characteristic of BLAKE-256, terminated at round 10.5, has $4+1+24=29$ unfixed conditions $\left(\Delta^{b} V^{6.5} \rightarrow \Delta^{t} V^{10.5}\right)$. For the top characteristic of BLAKE-256, if we choose the active bit position $y=20 \in \mathbb{X}_{256}$, which is also the case of [12], there should be 71 unfixed conditions and the complexity of this 8-round boomerang attack is $2^{(29+71) \times 2}=2^{200}$. However, if we choose $y=28 \in \mathbb{X}_{256}, 1$ condition in $\Delta^{t} V^{3} \rightarrow \Delta^{t} V^{2.5}$ can be eliminated and the complexity of the attack can lower to $2^{(29+70) \times 2}=2^{198}$.

BLAKE2s: Similar to BLAKE2b, the bottom characteristic for BLAKE2s, terminated at round 10, has $4+1=5$ unfixed conditions. The top characteristic has 88 unfixed conditions. So the complexity of this 7.5-round boomerang attack for BLAKE2s is $2^{(5+88) \times 2}=2^{186}$. Like BLAKE-256, if we choose $y=28 \in \mathbb{X}_{2 s}$, we can eliminate 1 condition in $\Delta^{t} V^{3} \rightarrow \Delta^{t} V^{2.5}$ and lower the complexity by $2^{2}$ to $2^{184}$.

Practical Verifications. For each member of BLAKE and BLAKE2, we present a 6.5 round ( from round 3.5 to round 10) Type I boomerang quartet based on our characteristics and present it in Appendix B In order to show the structural difference between BLAKE and BLAKE2, we use the examples with the same message difference, which means: for BLAKE-256 and BLAKE2s, $\Delta^{t} m_{5}=(28)\left(y=28 \in \mathbb{X}_{256} \bigcap \mathbb{X}_{2 s}\right)$ and $\Delta^{b} m_{11}=(31)$; for BLAKE-512 and BLAKE2b, $\Delta^{t} m_{5}=(9)\left(y=9 \in \mathbb{X}_{512} \bigcap \mathbb{X}_{2 b}\right)$ and $\Delta^{b} m_{11}=(63)$. 


\section{Conclusion}

In this paper, we compare the security margin of BLAKE and BLAKE2 under the boomerang attack model. We deduce valid differential characteristics and present boomerang attacks on keyed permutations of BLAKE-512, BLAKE2b, BLAKE-256 and BLAKE2s. According to our analysis, the boomerang method can mount to similar rounds for BLAKE and BLAKE2. For the same number of rounds, the complexities for attacking BLAKE2 are slightly higher than those for BLAKE, which indicates that some tweaks introduced by BLAKE2, aiming at enhancing efficiency and flexibility, have accidentally reinforced the resistance against the boomerang attack. However, since BLAKE has more rounds than BLAKE2, the security margin of BLAKE is still higher than that of BLAKE2. This result is in accordance with the assumptions of the designers.

Acknowledgement. This work has been supported by the National Natural Science Foundation of China (Grant No. 61133013) and by 973 Program (Grant No. 2013CB834205).

\section{References}

1. Wang, X., Yu, H.: How to break md5 and other hash functions. In: Advances in Cryptology-EUROCRYPT 2005. Springer (2005) 19-35

2. Wang, X., Yin, Y.L., Yu, H.: Finding collisions in the full sha-1. In: Advances in Cryptology-CRYPTO 2005, Springer (2005) 17-36

3. Bertoni, G., Daemen, J., Peeters, M., Assche, G.: The keccak reference. Submission to NIST (Round 3) 13 (2011)

4. Aumasson, J.P., Henzen, L., Meier, W., Phan, R.C.W.: Sha-3 proposal blake. Submission to NIST (2008)

5. Chang, S.j., Perlner, R., Burr, W.E., Turan, M.S., Kelsey, J.M., Paul, S., Bassham, L.E.: Third-round report of the SHA-3 cryptographic hash algorithm competition. Citeseer (2012)

6. Aumasson, J.P., Neves, S., Wilcox-OHearn, Z., Winnerlein, C.: Blake2: simpler, smaller, fast as md5. In: Applied Cryptography and Network Security, Springer (2013) 119-135

7. Aumasson, J.P., Guo, J., Knellwolf, S., Matusiewicz, K., Meier, W.: Differential and invertibility properties of blake. In: Fast Software Encryption, Springer (2010) 318-332

8. Dunkelman, O., Khovratovich, D.: Iterative differentials, symmetries, and message modification in blake-256. In: ECRYPT2 Hash Workshop. Volume 2011. (2011)

9. Ji, L., Liangyu, X.: Attacks on round-reduced blake. Technical report, Citeseer (2009)

10. Biryukov, A., Nikolić, I., Roy, A.: Boomerang attacks on blake-32. In: Fast Software Encryption, Springer (2011) $218-237$

11. Leurent, G.: Arxtools: A toolkit for arx analysis. In: The Third SHA-3 Candidate Conference. (2012)

12. Bai, D., Yu, H., Wang, G., Wang, X.: Improved boomerang attacks on round-reduced sm3 and blake-256. (2013) http://eprint.iacr.org/.

13. Guo, J., Karpman, P., Nikolić, I., Wang, L., Wu, S.: Analysis of blake2. In: Topics in Cryptology-CT-RSA 2014. Springer (2014) 402-423

14. Wagner, D.: The boomerang attack. In: Fast Software Encryption, Springer (1999) 156-170

15. Kelsey, J., Kohno, T., Schneier, B.: Amplified boomerang attacks against reduced-round mars and serpent. In: Fast Software Encryption, Springer (2001) 75-93

16. Biham, E., Dunkelman, O., Keller, N.: The rectangle attackrectangling the serpent. In: Advances in CryptologyEUROCRYPT 2001. Springer (2001) 340-357

17. Biham, E., Dunkelman, O., Keller, N.: Related-key boomerang and rectangle attacks. In: Advances in CryptologyEUROCRYPT 2005. Springer (2005) 507-525

18. Lamberger, M., Mendel, F.: Higher-order differential attack on reduced sha-256. IACR Cryptology ePrint Archive 2011 (2011) 37

19. Biryukov, A., Lamberger, M., Mendel, F., Nikolić, I.: Second-order differential collisions for reduced sha-256. In: Advances in Cryptology-ASIACRYPT 2011. Springer (2011) 270-287

20. Mendel, F., Nad, T.: Boomerang distinguisher for the simd-512 compression function. In: Progress in CryptologyINDOCRYPT 2011. Springer (2011) 255-269

21. Sasaki, Y., Wang, L., Takasaki, Y., Sakiyama, K., Ohta, K.: Boomerang distinguishers for full has-160 compression function. In: Advances in Information and Computer Security. Springer (2012) 156-169 
22. Sasaki, Y., Wang, L.: Distinguishers beyond three rounds of the ripemd-128/-160 compression functions. In: Applied Cryptography and Network Security, Springer (2012) 275-292

23. Leurent, G., Roy, A.: Boomerang attacks on hash function using auxiliary differentials. In: Topics in CryptologyCT-RSA 2012. Springer (2012) 215-230

24. Yu, H., Chen, J., Wang, X.: The boomerang attacks on the round-reduced skein-512. In: Selected Areas in Cryptography, Springer (2013) 287-303

25. Kircanski, A., Shen, Y., Wang, G., Youssef, A.M.: Boomerang and slide-rotational analysis of the sm3 hash function. In: Selected Areas in Cryptography, Springer (2013) 304-320

26. Bai, D., Yu, H., Wang, G., Wang, X.: Improved boomerang attacks on sm3. In: Information Security and Privacy, Springer (2013) 251-266

27. Wagner, D.: A generalized birthday problem. In: Advances in cryptologyCRYPTO 2002. Springer (2002) 288-304

\section{Appendix}

\section{A The Bottom \& Top Differential Characteristics for BLAKE and BLAKE2}

Table 4. The bottom characteristic for BLAKE-512. $\Delta^{b} m_{11}=(63)$.

\begin{tabular}{|c|c|c|c|c|c|}
\hline Variable & Difference (Numeric Form) & Cond & Variable & Difference (Numeric Form) & Cond \\
\hline$\Delta^{b} V^{6.5}$ & $\begin{aligned} \Delta^{b} v_{0}^{6.5} & =(63,42,35,10,3) \\
\Delta^{b} v_{1}^{6.5} & =(63,31) \\
\Delta^{b} v_{2}^{6.5} & =(63,47,24,15) \\
\Delta^{b} v_{3}^{6.5} & =(60,56,40,31,24,10,8) \\
\Delta^{b} v_{4}^{6.5} & =(60,56,47,40,35,24,15,8) \\
\Delta^{b} v_{5}^{6.5} & =(63,35,24,3) \\
\Delta^{b} v_{7}^{6.5} & =(63,47,24,15) \\
\Delta^{b} v_{8}^{6.5} & =(63,47,31,15) \\
\Delta^{b} v_{10}^{6.5} & =(24) \\
\Delta^{b} v_{11}^{6.5} & =(63,31) \\
\Delta^{b} v_{13}^{6.5} & =(63) \\
\Delta^{b} v_{14}^{6.5} & =(35,31,10) \\
\Delta^{b} v_{15}^{6.5} & =(56,42,31,24,10)\end{aligned}$ & - & \multirow[t]{2}{*}{$\Delta^{b} V^{10.5}$} & $\begin{aligned} \Delta^{b} v_{0}^{10.5} & =(63,6) \\
\Delta^{b} v_{1}^{10.5} & =(43,36,11) \\
\Delta^{b} v_{2}^{10.5} & =(22) \\
\Delta^{b} v_{3}^{10.5} & =(54) \\
\Delta^{b} v_{4}^{10.5} & =(59,43,36,20,4) \\
\Delta^{b} v_{5}^{10.5} & =(57,48,41,32,16,9,0) \\
\Delta^{b} v_{6}^{10.5} & =(59,36,11) \\
\Delta^{b} v_{7}^{10.5} & =(52,43,27,4) \\
\Delta^{b} v_{8}^{10.5} & =(54,47,31,15) \\
\Delta^{b} v_{9}^{10.5} & =(59,52,27,20,4) \\
\Delta^{b} v_{10}^{10.5} & =(47,6) \\
\Delta^{b} v_{11}^{10.5} & =(63,38,15) \\
\Delta^{b} v_{12}^{10.5} & =(54,47,15)\end{aligned}$ & \multirow[t]{2}{*}{24} \\
\hline & $\begin{array}{l}\Delta^{b} v_{0}^{7}=(63,24) \\
\Delta^{b} v_{1}^{7}=(63,31) \\
\Delta^{b} v_{2}^{7}=(63)\end{array}$ & & & $\begin{array}{l}\Delta^{b} v_{13}^{10.5}=(59,52,27,20) \\
\Delta^{b} v_{14}^{10.5}=(6) \\
\Delta^{b} v_{15}^{10.5}=(63,38)\end{array}$ & \\
\hline$\Delta^{b} V^{7}$ & $\begin{array}{l}\Delta^{b} v_{4}^{7}=(63,24) \\
\Delta^{b} v_{5}^{7}=(63,31) \\
\Delta^{b} v_{8}^{7}=(63) \\
\Delta^{b} v_{9}^{7}=(63,47,31,15) \\
\Delta^{b} v_{10}^{7}=(63) \\
\Delta^{b} v_{13}^{7}=(47,15) \\
\Delta^{b} v_{14}^{7}=(63,31)\end{array}$ & $\begin{array}{c}40 \\
(40 \text { fixed })\end{array}$ & \multirow{5}{*}{$\Delta^{b} V^{11}$} & $\begin{array}{l}\Delta^{b} v_{0}^{11}=(63,57,48,41,22,16,13,9,6) \\
\Delta^{b} v_{1}^{11}=(63,61,59,43,38,34,22,13,11,2) \\
\Delta^{b} v_{2}^{11}=(54,52,50,27,18,11,6,4) \\
\Delta^{b} v_{3}^{11}=(61,59,54,50,36,20,18,13,4) \\
\Delta^{b} v_{4}^{11}=(59,57,55,39,34,23,9,7,2) \\
\Delta^{b} v_{5}^{11}=(59,50,27,14,2) \\
\Delta^{b} v_{6}^{11}=(55,39,34,32,23,20,11,7,2,0)\end{array}$ & \multirow{5}{*}{138} \\
\hline$\Delta^{b} V^{7.5}$ & $\begin{array}{l}\Delta^{b} v_{2}^{7.5}=(63) \\
\Delta^{b} v_{8}^{7.5}=(63) \\
\Delta^{b} v_{13}^{7.5}=(63,31)\end{array}$ & $\begin{array}{c}6 \\
(2 \text { fixed })\end{array}$ & & $\begin{aligned} \Delta^{b} v_{7}^{11}= & (59,55,39,36,32,25,23 \\
& 20,16,11,9,7,4) \\
\Delta^{b} v_{8}^{11}= & (54,47,36,34,31,27,20,15,11,2)\end{aligned}$ & \\
\hline$\Delta^{b} V^{8}$ & $\Delta^{b} v_{2}^{8}=(63)$ & 0 & & $\Delta^{b} v_{9}^{11}=(61,45,43,34,20,6,4,2)$ & \\
\hline $\begin{array}{l}\Delta^{b} V^{8.5} \cdots \\
\cdots \Delta^{b} V^{9.5}\end{array}$ & $\phi$ & 0 & & $\begin{array}{l}\Delta^{b} v_{10}^{11}=(61,38,32,25,22,6,0) \\
\Delta^{b} v_{11}^{11}=(61,50,45,43,38,31,18)\end{array}$ & \\
\hline$\Delta^{b} V^{10}:$ & $\begin{array}{l}\Delta^{b} v_{0}^{10}=(63) \\
\Delta^{b} v_{5}^{10}=(36) \\
\Delta^{b} v_{10}^{10}=(47) \\
\Delta^{b} v_{15}^{10}=(47)\end{array}$ & 1 & & $\begin{array}{l}\Delta^{b} v_{12}^{11}=(63,61,50,47,45,43,31,27,22,18,11) \\
\Delta^{b} v_{13}^{11}=(54,52,34,20,2) \\
\Delta^{b} v_{14}^{11}=(61,59,45,43,38,36,34,22,11,6,4,2) \\
\Delta^{b} v_{15}^{11}=(61,48,47,41,22,16,9,6)\end{array}$ & \\
\hline
\end{tabular}


Table 5. The bottom characteristic for BLAKE2b. $\Delta^{b} m_{11}=(63)$.

\begin{tabular}{|c|c|c|c|c|c|}
\hline Variable & Difference (Numeric Form) & Cond & Variable & Difference (Numeric Form) & Cond \\
\hline$\Delta^{b} V^{6.5}$ & $\begin{array}{l}\Delta^{b} v_{0}^{6.5}=(63,62,54,30,22) \\
\Delta^{b} v_{1}^{6.5}=(63,31) \\
\Delta^{b} v_{2}^{6.5}=(63,47,23,15) \\
\Delta^{b} v_{3}^{6.5}=(62,55,46,39,31,23,7) \\
\Delta^{b} v_{4}^{6.5}=(55,47,46,39,23,22,15,7) \\
\Delta^{b} v_{5}^{6.5}=(63,54,23,22) \\
\Delta^{b} v_{7}^{6.5}=(63,47,23,15) \\
\Delta^{b} v_{8}^{6.5}=(63,47,31,15) \\
\Delta^{b} v_{10}^{6.5}=(23) \\
\Delta^{b} v_{11}^{6.5}=(63,31) \\
\Delta^{b} v_{13}^{6.5}=(63) \\
\Delta^{b} v_{14}^{6.5}=(62,31,22) \\
\Delta^{b} v_{15}^{6.5}=(62,55,31,30,23) \\
\Delta^{b} v_{0}^{7}=(63,23) \\
\Delta^{b} v_{1}^{7}=(63,31) \\
\Delta^{b} v_{2}^{7}=(63)\end{array}$ & - & $\Delta^{b} V^{10.5}:$ & $\begin{array}{l}\Delta^{b} v_{0}^{10.5}=(63,7) \\
\Delta^{b} v_{1}^{10.5}=(56,48,24) \\
\Delta^{b} v_{2}^{10.5}=(23) \\
\Delta^{b} v_{3}^{10.5}=(55) \\
\Delta^{b} v_{4}^{10.5}=(56,48,32,16,8) \\
\Delta^{b} v_{5}^{10.5}=(57,41,33,25,17,9,1) \\
\Delta^{b} v_{6}^{10.5}=(48,24,8) \\
\Delta^{b} v_{7}^{10.5}=(56,40,16,0) \\
\Delta^{b} v_{8}^{10.5}=(55,47,31,15) \\
\Delta^{b} v_{9}^{10.5}=(40,32,16,8,0) \\
\Delta^{b} v_{10}^{10.5}=(47,7) \\
\Delta^{b} v_{11}^{10.5}=(63,39,15) \\
\Delta^{b} v_{12}^{10.5}=(55,47,15) \\
\Delta^{b} v_{13}^{10.5}=(40,32,8,0) \\
\Delta^{b} v_{14}^{10.5}=(7) \\
\Delta^{b} v_{15}^{10.5}=(63,39)\end{array}$ & 24 \\
\hline$\Delta^{b} V^{7}$ & $\begin{array}{l}\Delta^{b} v_{4}^{7}=(63,23) \\
\Delta^{b} v_{5}^{7}=(63,31) \\
\Delta^{b} v_{8}^{7}=(63) \\
\Delta^{b} v_{9}^{7}=(63,47,31,15) \\
\Delta^{b} v_{10}^{7}=(63) \\
\Delta^{b} v_{13}^{7}=(47,15) \\
\Delta^{b} v_{14}^{7}=(63,31)\end{array}$ & $\begin{array}{c}40 \\
(40 \text { fixed })\end{array}$ & \multirow{5}{*}{$\Delta^{b} V^{11}$} & $\begin{aligned} \Delta^{b} v_{0}^{11} & =(63,41,33,23,17,15,9,7,1) \\
\Delta^{b} v_{1}^{11} & =(56,48,39,24,23,16,15,8) \\
\Delta^{b} v_{2}^{11} & =(55,40,32,24,16,7) \\
\Delta^{b} v_{3}^{11} & =(63,55,48,16,15,8,0) \\
\Delta^{b} v_{4}^{11} & =(49,48,16,8,1) \\
\Delta^{b} v_{5}^{11} & =(50,40,16,8,0) \\
\Delta^{b} v_{6}^{11} & =(57,49,48,33,32,25,24,17,16,1)\end{aligned}$ & \multirow{5}{*}{124} \\
\hline$\Delta^{b} V^{7.5}$ & $\begin{array}{l}\Delta^{b} v_{2}^{7.5}=(63) \\
\Delta^{b} v_{8}^{7.5}=(63) \\
\Delta^{b} v_{13}^{7.5}=(63,31)\end{array}$ & $\begin{array}{c}6 \\
(2 \text { fixed })\end{array}$ & & $\begin{aligned} \Delta^{b} v_{7}^{11} & =(57,48,41,32,24,17,16,8,1) \\
\Delta^{b} v_{8}^{11} & =(55,47,40,32,31,24,16,15) \\
\Delta^{b} v_{9}^{11} & =(63,56,48,47,32,7)\end{aligned}$ & \\
\hline$\Delta^{b} V^{8}$ & $\Delta^{b} v_{2}^{8}=(63)$ & 0 & & $\Delta^{b} v_{10}^{11}=(63,57,49,39,25,23,7)$ & \\
\hline $\begin{array}{l}\Delta^{b} V^{8.5} \cdots \\
\cdots \Delta^{b} V^{9.5}\end{array}$ & $\phi$ & 0 & & $\begin{array}{l}\Delta^{b} v_{11}^{11}=(63,56,47,39,32,31,0) \\
\Delta^{b} v_{12}^{11}=(56,40,32,31,24,23,0)\end{array}$ & \\
\hline$\Delta^{b} V^{10}:$ & $\begin{array}{l}\Delta^{b} v_{0}^{10}=(63) \\
\Delta^{b} v_{5}^{10}=(48) \\
\Delta^{b} v_{10}^{10}=(47) \\
\Delta^{b} v_{15}^{10}=(47)\end{array}$ & 1 & & $\begin{array}{l}\Delta^{b} v_{13}^{11}=(55,48,32,16,0) \\
\Delta^{b} v_{14}^{11}=(63,56,47,39,24,23,8,7) \\
\Delta^{b} v_{15}^{11}=(63,47,41,33,23,9,7,1)\end{array}$ & \\
\hline
\end{tabular}


Table 6. The bottom characteristic for BLAKE-256. $\Delta^{b} m_{11}=(31)$.

\begin{tabular}{|c|c|c|c|c|c|}
\hline Variable & Difference (Numeric Form) & Cond & Variable & Difference (Numeric Form) & Cond \\
\hline \multirow[b]{4}{*}{$\Delta^{b} V^{6.5}$} & \multirow{4}{*}{$\begin{aligned} \Delta^{b} v_{0}^{6.5} & =(31,22,18,6,2) \\
\Delta^{b} v_{1}^{6.5} & =(31,15) \\
\Delta^{b} v_{2}^{6.5} & =(31,23,11,7) \\
\Delta^{b} v_{3}^{6.5} & =(30,27,19,15,11,6,3) \\
\Delta^{b} v_{4}^{6.5} & =(30,27,23,19,18,11,7,3) \\
\Delta^{b} v_{5}^{6.5} & =(31,18,11,2) \\
\Delta^{b} v_{7}^{6.5} & =(31,23,11,7) \\
\Delta^{b} v_{8}^{6.5} & =(31,23,15,7) \\
\Delta^{b} v_{10}^{6.5} & =(11) \\
\Delta^{b} v_{11}^{6.5} & =(31,15) \\
\Delta^{b} v_{13}^{6.5} & =(31) \\
\Delta^{b} v_{14}^{6.5} & =(18,15,6) \\
\Delta^{b} v_{15}^{6.5} & =(27,22,15,11,6)\end{aligned}$} & \multirow[b]{4}{*}{ - } & $\Delta^{b} V^{8}$ & $\Delta^{b} v_{2}^{8}=(31)$ & 0 \\
\hline & & & $\Delta^{b} V^{8.5} \cdots \Delta^{b} V^{9.5}$ & $\phi$ & 0 \\
\hline & & & $\Delta^{b} V^{10}:$ & $\begin{array}{l}\Delta^{b} v_{0}^{10}=(31) \\
\Delta^{b} v_{5}^{10}=(16) \\
\Delta^{b} v_{10}^{10}=(23) \\
\Delta^{b} v_{15}^{10}=(23)\end{array}$ & 1 \\
\hline & & & \multirow{3}{*}{$\Delta^{b} V^{10.5}:$} & \multirow{3}{*}{$\begin{aligned} \Delta^{b} v_{0}^{10.5} & =(31,3) \\
\Delta^{b} v_{1}^{10.5} & =(20,16,4) \\
\Delta^{b} v_{2}^{10.5} & =(11) \\
\Delta^{b} v_{3}^{10.5} & =(27) \\
\Delta^{b} v_{4}^{10.5} & =(28,20,16,8,0) \\
\Delta^{b} v_{5}^{10.5} & =(29,25,21,17,13,5,1) \\
\Delta^{b} v_{6}^{10.5} & =(28,16,4) \\
\Delta^{b} v_{7}^{10.5} & =(24,20,12,0) \\
\Delta^{b} v_{8}^{10.5} & =(27,23,15,7) \\
\Delta^{b} v_{9}^{10.5} & =(28,24,12,8,0) \\
\Delta^{b} v_{10}^{10.5} & =(23,3) \\
\Delta^{b} v_{11}^{10.5} & =(31,19,7) \\
\Delta^{b} v_{12}^{10.5} & =(27,23,7) \\
\Delta^{b} v_{13}^{10.5} & =(28,24,12,8) \\
\Delta^{b} v_{14}^{10.5} & =(3) \\
\Delta^{b} v_{15}^{10.5} & =(31,19)\end{aligned}$} & \multirow{3}{*}{24} \\
\hline$\Delta^{b} V^{7}$ & $\begin{array}{l}\Delta^{b} v_{0}^{7}=(31,11) \\
\Delta^{b} v_{1}^{7}=(31,15) \\
\Delta^{b} v_{2}^{7}=(31) \\
\Delta^{b} v_{4}^{7}=(31,11) \\
\Delta^{b} v_{5}^{7}=(31,15) \\
\Delta^{b} v_{8}^{7}=(31) \\
\Delta^{b} v_{9}^{7}=(31,23,15,7) \\
\Delta^{b} v_{10}^{7}=(31) \\
\Delta^{b} v_{13}^{7}=(23,7) \\
\Delta^{b} v_{14}^{7}=(31,15)\end{array}$ & 40 (40 fixed) & & & \\
\hline$\Delta^{b} V^{7.5}$ & $\begin{array}{l}\Delta^{b} v_{2}^{7.5}=(31) \\
\Delta^{b} v_{8}^{7.5}=(31) \\
\Delta^{b} v_{13}^{7.5}=(31,15)\end{array}$ & 6 (2 fixed) & & & \\
\hline
\end{tabular}

Table 7. The bottom characteristic for BLAKE2s. $\Delta^{b} m_{11}=(31)$.

\begin{tabular}{|c|c|c|c|c|c|}
\hline Variable & Difference (Numeric Form) & Cond & Variable & Difference (Numeric Form) & Cond \\
\hline \multirow{5}{*}{$\Delta^{b} V^{6.5}$} & \multirow{5}{*}{$\begin{array}{l}\Delta^{b} v_{0}^{6.5}=(31,22,18,6,2) \\
\Delta^{b} v_{1}^{6.5}=(31,15) \\
\Delta^{b} v_{2}^{6.5}=(31,23,11,7) \\
\Delta^{b} v_{3}^{6.5}=(30,27,19,15,11,6,3) \\
\Delta^{b} v_{4}^{6.5}=(30,27,23,19,18,11,7,3) \\
\Delta^{b} v_{5}^{6.5}=(31,18,11,2) \\
\Delta^{b} v_{7}^{6.5}=(31,23,11,7) \\
\Delta^{b} v_{8}^{6.5}=(31,23,15,7) \\
\Delta^{b} v_{10}^{6.5}=(11) \\
\Delta^{b} v_{11}^{6.5}=(31,15) \\
\Delta^{b} v_{13}^{6.5}=(31) \\
\Delta^{b} v_{14}^{6.5}=(18,15,6) \\
\Delta^{b} v_{15}^{6.5}=(27,22,15,11,6)\end{array}$} & \multirow{5}{*}{1} & $\Delta^{b} V^{7}$ & $\begin{array}{l}\Delta^{b} v_{0}^{7}=(31,11) \\
\Delta^{b} v_{1}^{7}=(31,15) \\
\Delta^{b} v_{2}^{7}=(31) \\
\Delta^{b} v_{4}^{7}=(31,11) \\
\Delta^{b} v_{5}^{7}=(31,15) \\
\Delta^{b} v_{8}^{7}=(31) \\
\Delta^{b} v_{9}^{7}=(31,23,15,7) \\
\Delta^{b} v_{10}^{7}=(31) \\
\Delta^{b} v_{13}^{7}=(23,7) \\
\Delta^{b} v_{14}^{7}=(31,15)\end{array}$ & 40 (40 fixed) \\
\hline & & & $\Delta^{b} V^{7.5}$ & $\begin{array}{l}\Delta^{b} v_{2}^{7.5}=(31) \\
\Delta^{b} v_{8}^{7.5}=(31) \\
\Delta^{b} v_{13}^{7.5}=(31,15)\end{array}$ & 6 (2 fixed) \\
\hline & & & $\Delta^{b} V^{8}$ & $\Delta^{b} v_{2}^{8}=(31)$ & 0 \\
\hline & & & $\Delta^{b} V^{8.5} \cdots \Delta^{b} V^{9.5}$ & $\phi$ & 0 \\
\hline & & & $\Delta^{b} V^{10}:$ & $\begin{array}{l}\Delta^{b} v_{0}^{10}=(31) \\
\Delta^{b} v_{5}^{10}=(16) \\
\Delta^{b} v_{10}^{10}=(23) \\
\Delta^{b} v_{15}^{10}=(23)\end{array}$ & 1 \\
\hline
\end{tabular}


Table 8. The top characteristic for BLAKE-512. Message difference is $\Delta^{t} m_{5}=(y)$ where $y \in \mathbb{X}_{512}$

\begin{tabular}{|c|c|c|}
\hline \multicolumn{3}{|c|}{$\mathbb{X}_{512}=\{5,9,18,20,22,29,34,38,41,45,48,52,54\}$} \\
\hline Variable & Difference (Numeric Form) & Cond \\
\hline$\Delta^{t} V^{2.5}:$ & $\begin{array}{l}\Delta^{t} v_{0}^{2.5}=(y+32) \\
\Delta^{t} v_{1}^{2.5}=(y+48, y+25, y+16) \\
\Delta^{t} v_{2}^{2.5}=(y+41, y+25, y+11, y+9, y+61, y+57) \\
\Delta^{t} v_{3}^{2.5}=(y+43, y+36, y+25, y+11, y+4) \\
\Delta^{t} v_{4}^{2.5}=(y+36, y+4) \\
\Delta^{t} v_{5}^{2.5}=(y) \\
\Delta^{t} v_{6}^{2.5}=(y+48, y+25, y+16, y) \\
\Delta^{t} v_{7}^{2.5}=(y+48, y+41, y+36, y+32, y+25, y+16, y+9, y, y+61, y+57) \\
\Delta^{t} v_{8}^{2.5}=(y+48, y+32, y+16, y) \\
\Delta^{t} v_{9}^{2.5}=(y+25) \\
\Delta^{t} v_{10}^{2.5}=(y+32, y+16) \\
\Delta^{t} v_{11}^{2.5}=(y+48, y+32, y+16) \\
\Delta^{t} v_{12}^{2.5}=(y+32) \\
\Delta^{t} v_{13}^{2.5}=(y+48, y+36, y+32, y+16, y+11, y) \\
\Delta^{t} v_{14}^{2.5}=(y+43, y+25, y+11, y+57) \\
\Delta^{t} v_{15}^{2.5}=(y+48)\end{array}$ & 51 \\
\hline$\Delta^{t} V^{3}$ & $\begin{array}{l}\Delta^{t} v_{0}^{3}=(y+32, y) \\
\Delta^{t} v_{3}^{3}=(y+25) \\
\Delta^{t} v_{4}^{3}=(y+32, y) \\
\Delta^{t} v_{7}^{3}=(y+25, y) \\
\Delta^{t} v_{8}^{3}=(y+48, y+32, y+16, y) \\
\Delta^{t} v_{11}^{3}=(y) \\
\Delta^{t} v_{12}^{3}=(y+48, y+16) \\
\Delta^{t} v_{15}^{3}=(y)\end{array}$ & 11 \\
\hline$\Delta^{t} V^{3.5}$ & $\begin{array}{l}\Delta^{t} v_{11}^{3.5}=(y) \\
\Delta^{t} v_{12}^{3.5}=(y+32, y)\end{array}$ & 2 \\
\hline$\Delta^{t} V^{4}$ & $\Delta^{t} v_{1}^{4}=(y)$ & 1 \\
\hline$\Delta^{t} V^{4.5} \cdots \Delta^{t} V^{5.5}$ & $\phi$ & 2 (2 fixed) \\
\hline$\Delta^{t} V^{6}$ & $\begin{array}{l}\Delta^{t} v_{1}^{6}=(y) \\
\Delta^{t} v_{6}^{6}=(y+37) \\
\Delta^{t} v_{11}^{6}=(y+48) \\
\Delta^{t} v_{12}^{6}=(y+48)\end{array}$ & 30 (29 fixed) \\
\hline$\Delta^{t} V^{6.5}$ & $\begin{array}{l}\Delta^{t} v_{0}^{6.5}=(y, y+55) \\
\Delta^{t} v_{1}^{6.5}=(y+7, y) \\
\Delta^{t} v_{2}^{6.5}=(y+44, y+37, y+12) \\
\Delta^{t} v_{3}^{6.5}=(y+23) \\
\Delta^{t} v_{4}^{6.5}=(y+53, y+44, y+37, y+28, y+5) \\
\Delta^{t} v_{5}^{6.5}=(y+44, y+37, y+21, y+5, y+60) \\
\Delta^{t} v_{6}^{6.5}=(y+49, y+42, y+33, y+17, y+10, y+1, y+58) \\
\Delta^{t} v_{7}^{6.5}=(y+37, y+12, y+60) \\
\Delta^{t} v_{8}^{6.5}=(y+48, y+39, y+16, y) \\
\Delta^{t} v_{9}^{6.5}=(y+48, y+32, y+16, y+55) \\
\Delta^{t} v_{10}^{6.5}=(y+53, y+28, y+21, y+5, y+60) \\
\Delta^{t} v_{11}^{6.5}=(y+48, y+7) \\
\Delta^{t} v_{12}^{6.5}=(y+48, y+39, y) \\
\Delta^{t} v_{13}^{6.5}=(y+48, y+16, y+55) \\
\Delta^{t} v_{14}^{6.5}=(y+53, y+28, y+21, y+60) \\
\Delta^{t} v_{15}^{6.5}=(y+7)\end{array}$ & - \\
\hline
\end{tabular}


Table 9. The top characteristic for BLAKE2b. Message difference is $\Delta^{t} m_{5}=(y)$ where $y \in \mathbb{X}_{2 b}$

\begin{tabular}{|c|c|c|}
\hline \multicolumn{3}{|c|}{$\begin{array}{c}\mathbb{X}_{2 b}=\{0,1,2,3,4,8,9,10,11,12,16,17,18,19,20,24,25,26,27,28 \\
32,33,34,35,36,40,41,42,43,44,48,49,50,51,52,56,57,58,59,60\}\end{array}$} \\
\hline Variable & Difference (Numeric Form) & Cond \\
\hline$\Delta^{t} V^{2.5}:$ & $\begin{array}{l}\Delta^{t} v_{0}^{2.5}=(y+32) \\
\Delta^{t} v_{1}^{2.5}=(y+48, y+24, y+16, y+1) \\
\Delta^{t} v_{2}^{2.5}=(y+47, y+40, y+33, y+24, y+8, y+1, y+63, y+56) \\
\Delta^{t} v_{3}^{2.5}=(y+31, y+25, y+24, y+23, y+63, y+55) \\
\Delta^{t} v_{4}^{2.5}=(y+25, y+23, y+1, y+55) \\
\Delta^{t} v_{5}^{2.5}=(y) \\
\Delta^{t} v_{6}^{2.5}=(y+48, y+24, y+16, y) \\
\Delta^{t} v_{7}^{2.5}=(y+48, y+47, y+40, y+33, y+32, y+24, y+23, y+16, y+8, y+1, y, y+56) \\
\Delta^{t} v_{8}^{2.5}=(y+49, y+48, y+33, y+32, y+17, y+16, y+1, y) \\
\Delta^{t} v_{9}^{2.5}=(y+24, y+1) \\
\Delta^{t} v_{10}^{2.5}=(y+32, y+16) \\
\Delta^{t} v_{11}^{2.5}=(y+48, y+32, y+16, y+1) \\
\Delta^{t} v_{12}^{2.5}=(y+33, y+32, y+1) \\
\Delta^{t} v_{13}^{2.5}=(y+49, y+48, y+32, y+23, y+17, y+16, y, y+63) \\
\Delta^{t} v_{14}^{2.5}=(y+31, y+24, y+1, y+63, y+56) \\
\Delta^{t} v_{15}^{2.5}=(y+48)\end{array}$ & 67 \\
\hline$\Delta^{t} V^{3}:$ & $\begin{array}{l}\Delta^{t} v_{0}^{3}=(y+32, y) \\
\Delta^{t} v_{1}^{3}=(y+1) \\
\Delta^{t} v_{3}^{3}=(y+24) \\
\Delta^{t} v_{4}^{3}=(y+32, y) \\
\Delta^{t} v_{7}^{3}=(y+24, y) \\
\Delta^{t} v_{8}^{3}=(y+48, y+32, y+16, y) \\
\Delta^{t} v_{9}^{3}=(y+1) \\
\Delta^{t} v_{11}^{3}=(y) \\
\Delta^{t} v_{12}^{3}=(y+48, y+16) \\
\Delta^{t} v_{13}^{3}=(y+33, y+1) \\
\Delta^{t} v_{15}^{3}=(y)\end{array}$ & 13 \\
\hline$\Delta^{t} V^{3.5}:$ & $\begin{array}{l}\Delta^{t} v_{1}^{3.5}=(y+1) \\
\Delta^{t} v_{11}^{3.5}=(y) \\
\Delta^{t} v_{12}^{3.5}=(y+32, y)\end{array}$ & 3 \\
\hline$\Delta^{t} V^{4}:$ & $\Delta^{t} v_{1}^{4}=(y)$ & 1 \\
\hline$\Delta^{t} V^{4.5} \cdots \Delta^{t} V^{5.5}:$ & $\phi$ & 2 (2 fixed) \\
\hline$\Delta^{t} V^{6}:$ & $\begin{array}{l}\Delta^{t} v_{1}^{6}=(y) \\
\Delta^{t} v_{6}^{6}=(y+49) \\
\Delta^{t} v_{11}^{6}=(y+48) \\
\Delta^{t} v_{12}^{6}=(y+48)\end{array}$ & 30 (29 fixed) \\
\hline$\Delta^{t} V^{6.5}:$ & $\begin{array}{l}\Delta^{t} v_{0}^{6.5}=(y, y+56) \\
\Delta^{t} v_{1}^{6.5}=(y+8, y) \\
\Delta^{t} v_{2}^{6.5}=(y+49, y+25, y+57) \\
\Delta^{t} v_{3}^{6.5}=(y+24) \\
\Delta^{t} v_{4}^{6.5}=(y+49, y+41, y+17, y+1, y+57) \\
\Delta^{t} v_{5}^{6.5}=(y+49, y+33, y+17, y+9, y+57) \\
\Delta^{t} v_{6}^{6.5}=(y+42, y+34, y+26, y+18, y+10, y+2, y+58) \\
\Delta^{t} v_{7}^{6.5}=(y+49, y+25, y+9) \\
\Delta^{t} v_{8}^{6.5}=(y+48, y+40, y+16, y) \\
\Delta^{t} v_{9}^{6.5}=(y+48, y+32, y+16, y+56) \\
\Delta^{t} v_{10}^{6.5}=(y+41, y+33, y+17, y+9, y+1) \\
\Delta^{t} v_{11}^{6.5}=(y+48, y+8) \\
\Delta^{t} v_{12}^{6.5}=(y+48, y+40, y) \\
\Delta^{t} v_{13}^{6.5}=(y+48, y+16, y+56) \\
\Delta^{t} v_{14}^{6.5}=(y+41, y+33, y+9, y+1) \\
\Delta^{t} v_{15}^{6.5}=(y+8)\end{array}$ & - \\
\hline
\end{tabular}


Table 10. The top characteristic for BLAKE-256. Message difference is $\Delta^{t} m_{5}=(y)$ where $y \in \mathbb{X}_{256}$.

\begin{tabular}{|c|c|c|}
\hline \multicolumn{3}{|c|}{$\mathbb{X}_{256}=\{20,28\}$} \\
\hline Variable & Difference (Numeric Form) & Cond \\
\hline$\Delta^{t} V^{2.5}:$ & 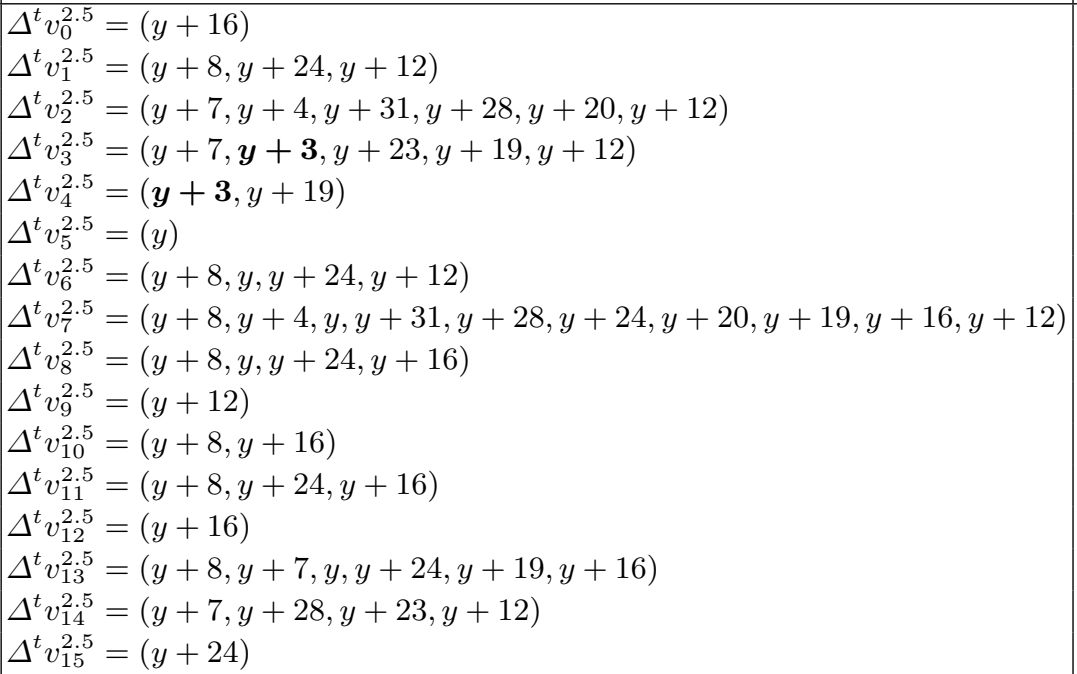 & $54 / 53^{*}$ \\
\hline$\Delta^{t} V^{3}$ & $\begin{array}{l}\Delta^{t} v_{0}^{3}=(y, y+16) \\
\Delta^{t} v_{3}^{3}=(y+12) \\
\Delta^{t} v_{4}^{3}=(y, y+16) \\
\Delta^{t} v_{7}^{3}=(y, y+12) \\
\Delta^{t} v_{8}^{3}=(y+8, y, y+24, y+16) \\
\Delta^{t} v_{11}^{3}=(y) \\
\Delta^{t} v_{12}^{3}=(y+8, y+24) \\
\Delta^{t} v_{15}^{3}=(y)\end{array}$ & 14 \\
\hline$\Delta^{t} V^{3.5}$ & $\begin{array}{l}\Delta^{t} v_{11}^{3.5}=(y) \\
\Delta^{t} v_{12}^{3.5}=(y, y+16)\end{array}$ & 2 \\
\hline$\Delta^{t} V^{4}$ & $\Delta^{t} v_{1}^{4}=(y)$ & 1 \\
\hline$\Delta^{t} V^{4.5} \cdots \Delta^{t} V^{5.5}$ & $\phi$ & 2 (2 fixed) \\
\hline$\Delta^{t} V^{6}$ & $\begin{array}{l}\Delta^{t} v_{1}^{6}=(y) \\
\Delta^{t} v_{6}^{6}=(y+17) \\
\Delta^{t} v_{11}^{6}=(y+24) \\
\Delta^{t} v_{12}^{6}=(y+24)\end{array}$ & 30 (29 fixed) \\
\hline$\Delta^{t} V^{6.5}$ & $\begin{array}{l}\Delta^{t} v_{0}^{6.5}=(y, y+28) \\
\Delta^{t} v_{1}^{6.5}=(y+4, y) \\
\Delta^{t} v_{2}^{6.5}=(y+5, y+21, y+17) \\
\Delta^{t} v_{3}^{6.5}=(y+12) \\
\Delta^{t} v_{4}^{6.5}=(y+1, y+25, y+21, y+17, y+13) \\
\Delta^{t} v_{5}^{6.5}=(y+9, y+1, y+29, y+21, y+17) \\
\Delta^{t} v_{6}^{6.5}=(y+6, y+2, y+30, y+26, y+22, y+18, y+14) \\
\Delta^{t} v_{7}^{6.5}=(y+5, y+29, y+17) \\
\Delta^{t} v_{8}^{6.5}=(y+8, y, y+24, y+20) \\
\Delta^{t} v_{9}^{6.5}=(y+8, y+28, y+24, y+16) \\
\Delta^{t} v_{10}^{6.5}=(y+9, y+1, y+29, y+25, y+13) \\
\Delta^{t} v_{11}^{6.5}=(y+4, y+24) \\
\Delta^{t} v_{12}^{6.5}=(y, y+24, y+20) \\
\Delta^{t} v_{13}^{6.5}=(y+8, y+28, y+24) \\
\Delta^{t} v_{14}^{6.5}=(y+9, y+29, y+25, y+13) \\
\Delta^{t} v_{15}^{6.5}=(y+4)\end{array}$ & - \\
\hline
\end{tabular}

*: If $y=28$, the condition $v_{3}^{2.5}[y+3]=\neg v_{4}^{2.5}[y+3]$ in $\Delta^{t} V^{3} \rightarrow \Delta^{t} V^{2.5}$ can be eliminated. 
Table 11. The top characteristic for BLAKE2s. Message difference is $\Delta^{t} m_{5}=(y)$ where $y \in \mathbb{X}_{2 s}$.

\begin{tabular}{|c|c|c|}
\hline \multicolumn{3}{|c|}{$\mathbb{X}_{2 s}=\{0,4,8,12,16,20,24,28\}$} \\
\hline Variable & Difference (Numeric Form) & Cond \\
\hline$\Delta^{t} V^{2.5}:$ & $\begin{array}{l}\Delta^{t} v_{0}^{2.5}=(y+16) \\
\Delta^{t} v_{1}^{2.5}=(y+8, y+1, y+24, y+12) \\
\Delta^{t} v_{2}^{2.5}=(y+7, y+4, y+1, y+31, y+28, y+20, y+17, y+12) \\
\Delta^{t} v_{3}^{2.5}=(y+7, \mathbf{y}+\mathbf{3}, y+23, y+19, y+13, y+12) \\
\Delta^{t} v_{4}^{2.5}=(\mathbf{y}+\mathbf{3}, y+1, y+19, y+13) \\
\Delta^{t} v_{5}^{2.5}=(y) \\
\Delta^{t} v_{6}^{2.5}=(y+8, y, y+24, y+12) \\
\Delta^{t} v_{7}^{2.5}=(y+8, y+4, y+1, y, y+31, y+28, y+24, y+20, y+19, y+17, y+16, y+12) \\
\Delta^{t} v_{8}^{2.5}=(y+9, y+8, y+1, y, y+25, y+24, y+17, y+16) \\
\Delta^{t} v_{9}^{2.5}=(y+1, y+12) \\
\Delta^{t} v_{10}^{2.5}=(y+8, y+16) \\
\Delta^{t} v_{11}^{2.5}=(y+8, y+1, y+24, y+16) \\
\Delta^{t} v_{12}^{2.5}=(y+1, y+17, y+16) \\
\Delta^{t} v_{13}^{2.5}=(y+9, y+8, y+7, y, y+25, y+24, y+19, y+16) \\
\Delta^{t} v_{14}^{2.5}=(y+7, y+1, y+28, y+23, y+12) \\
\Delta^{t} v_{15}^{2.5}=(y+24)\end{array}$ & $68 / 67^{*}$ \\
\hline$\Delta^{t} V^{3}:$ & $\begin{array}{l}\Delta^{t} v_{0}^{3}=(y, y+16) \\
\Delta^{t} v_{1}^{3}=(y+1) \\
\Delta^{t} v_{3}^{3}=(y+12) \\
\Delta^{t} v_{4}^{3}=(y, y+16) \\
\Delta^{t} v_{7}^{3}=(y, y+12) \\
\Delta^{t} v_{8}^{3}=(y+8, y, y+24, y+16) \\
\Delta^{t} v_{9}^{3}=(y+1) \\
\Delta^{t} v_{11}^{3}=(y) \\
\Delta^{t} v_{12}^{3}=(y+8, y+24) \\
\Delta^{t} v_{13}^{3}=(y+1, y+17) \\
\Delta^{t} v_{15}^{3}=(y)\end{array}$ & 16 \\
\hline$\Delta^{t} V^{3.5}:$ & $\begin{array}{l}\Delta^{t} v_{1}^{3.5}=(y+1) \\
\Delta^{t} v_{11}^{3.5}=(y) \\
\Delta^{t} v_{12}^{3.5}=(y, y+16)\end{array}$ & 3 \\
\hline$\Delta^{t} V^{4}:$ & $\Delta^{t} v_{1}^{4}=(y)$ & 1 \\
\hline$\Delta^{t} V^{4.5} \cdots \Delta^{t} V^{5.5}:$ & $\phi$ & 2 (2 fixed) \\
\hline$\Delta^{t} V^{6}:$ & $\begin{array}{l}\Delta^{t} v_{1}^{6}=(y) \\
\Delta^{t} v_{6}^{6}=(y+17) \\
\Delta^{t} v_{11}^{6}=(y+24) \\
\Delta^{t} v_{12}^{6}=(y+24)\end{array}$ & $30(29$ fixed $)$ \\
\hline$\Delta^{t} V^{6.5}:$ & $\begin{array}{l}\Delta^{t} v_{0}^{6.5}=(y, y+28) \\
\Delta^{t} v_{1}^{6.5}=(y+4, y) \\
\Delta^{t} v_{2}^{6.5}=(y+5, y+21, y+17) \\
\Delta^{t} v_{3}^{6.5}=(y+12) \\
\Delta^{t} v_{4}^{6.5}=(y+1, y+25, y+21, y+17, y+13) \\
\Delta^{t} v_{5}^{6.5}=(y+9, y+1, y+29, y+21, y+17) \\
\Delta^{t} v_{6}^{6.5}=(y+6, y+2, y+30, y+26, y+22, y+18, y+14) \\
\Delta^{t} v_{7}^{6.5}=(y+5, y+29, y+17) \\
\Delta^{t} v_{8}^{6.5}=(y+8, y, y+24, y+20) \\
\Delta^{t} v_{9}^{6.5}=(y+8, y+28, y+24, y+16) \\
\Delta^{t} v_{10}^{6.5}=(y+9, y+1, y+29, y+25, y+13) \\
\Delta^{t} v_{11}^{6.5}=(y+4, y+24) \\
\Delta^{t} v_{12}^{6.5}=(y, y+24, y+20) \\
\Delta^{t} v_{13}^{6.5}=(y+8, y+28, y+24) \\
\Delta^{t} v_{14}^{6.5}=(y+9, y+29, y+25, y+13) \\
\Delta^{t} v_{15}^{6.5}=(y+4)\end{array}$ & - \\
\hline
\end{tabular}

*: If $y=28$, the condition $v_{3}^{2.5}[y+3]=\neg v_{4}^{2.5}[y+3]$ in $\Delta^{t} V^{3} \rightarrow \Delta^{t} V^{2.5}$ can be eliminated. 


\section{B 6.5-Round Examples For BLAKE and BLAKE2}

The main difference between BLAKE-256 and BLAKE2s (BLAKE-512 and BLAKE2b) is at $\Delta^{t} v_{1}^{3.5}$, where $\Delta^{t} v_{1}^{3.5}=(29)$ for BLAKE-2s $\left(\Delta^{t} v_{1}^{3.5}=(10)\right.$ for BLAKE-2b) and $\phi$ for BLAKE-256 (BLAKE-512). We specifically emphasize this part with bold dark format.

Table 12. Example for 6.5-round BLAKE-256 with $y=28 \in \mathbb{X}_{256} \bigcap \mathbb{X}_{2 s}$.

\begin{tabular}{|c|c|c|c|c|c|c|c|c|}
\hline$\Delta M$ & \multicolumn{8}{|c|}{$\Delta^{b} m_{11}=(31), \Delta^{t} m_{5}=(28)$} \\
\hline \multirow{2}{*}{${ }_{a} M$} & $0 x 932 \mathrm{a} 5 \mathrm{~d} 7 \mathrm{f}$ & $0 \times a 2625330$ & 0x46a9466f & 0xae3052a3 & 0xbf9a6338 & $0 x d 4167790$ & 0x7bf0ef5e & 0x4ef572ba \\
\hline & $0 \times 30$ & $0 \times 23$ & $0 x 6$ & 0xa75b42e8 & $0 \times 3$ & 0xb56003b4 & $0 \times 7 a 4 d b 777$ & $0 \times 7$ \\
\hline \multirow{2}{*}{${ }_{b} M$} & $17 \mathrm{f}$ & $0 x a$ & $0 \times 4$ & $0 x a$ & 338 & 790 & $0 \mathrm{x}$ & $2 \mathrm{ba}$ \\
\hline & $0 \times 30$ & $0 \times 2$ & $0 \times 6 f$ & $42 \mathrm{e} 8$ & 0x3cb6d30e & 0xb56003b4 & $0 \times 7 \mathrm{a} 4 \mathrm{db} 777$ & $\mathrm{~b} 79 \mathrm{a}$ \\
\hline \multirow{2}{*}{${ }_{c} M$} & $7 \mathrm{f}$ & $0 x$ & $0 x$ & $0 \mathrm{x}$ & 38 & & f5e & \\
\hline & $0 \times 30$ & $0 \times 2$ & $0 \times 6$ & $0 \mathrm{x}$ & $30 \mathrm{e}$ & $\mathrm{b} 4$ & $0 \mathrm{x}^{\prime}$ & $79 a$ \\
\hline \multirow{2}{*}{${ }_{d} M$} & $0 \mathrm{x}$ & 0xa & $0 \mathrm{x}$ & & & & & \\
\hline & $0 \times 30$ & $0 \times 2$ & $0 \times 6 \mathrm{fb}$ & $0 \times 2$ & $0 \times 3 \mathrm{cb}$ & 3b4 & & $79 \mathrm{a}$ \\
\hline$\Delta^{t} V^{3.5}$ & \multicolumn{8}{|c|}{$\Delta^{\mathbf{t}} \mathbf{v}_{\mathbf{1}}^{\mathbf{3 . 5}}=\boldsymbol{\phi}, \Delta^{t} v_{11}^{3.5}=(28), \Delta^{t} v_{12}^{3.5}=(28,2)$} \\
\hline \multirow{2}{*}{${ }_{a} V^{3.5}$} & $0 \mathrm{x}^{7}$ & $\mathrm{~b}$ & $0 \times 7$ & 6 & $0 \mathrm{a} 0$ & b3 & 0x & $\mathrm{a} 2$ \\
\hline & $0 \times f^{2}$ & $0 \times 2 c$ & $0 \times 8$ & $\mathrm{f} 04$ & $29 f$ & 4 & $97 \mathrm{f}$ & $f 3$ \\
\hline \multirow{2}{*}{${ }_{b} V^{3.5}$} & 0xce & $0 x c 3$ & $0 x b$ & Oxbf & e46 & c9e & 5 a99 & $0 \times 2 e$ \\
\hline & $0 \times 89$ & $0 x f c 2$ & $0 x b c$ & $0 \times 5 a$ & 0xa42a029b & $0 \times 60$ & $62 \mathrm{de}$ & $\mathrm{d} 434$ \\
\hline \multirow{2}{*}{${ }_{c} V^{3.5}$} & $0 \times 7$ & $0 \times 5 f$ & Oxre & e6 & $\mathrm{a} 0 \mathrm{a} 0$ & $\mathrm{a} 3 \mathrm{~b} 3$ & $7 \mathrm{~d} 3 \mathrm{~b}$ & $0 \times 6$ \\
\hline & $0 x f 2$ & $0 \times 2 d$ & $0 \times 8$ & If04 & $629 f$ & ee4 & $197 \mathrm{f}$ & ef3 \\
\hline \multirow{2}{*}{${ }_{d} V^{3.5}$} & $0 \mathrm{xc}$ & $0 \mathrm{c} 1$ & $0 \mathrm{xb}$ & $0 \times b$ & e46 & $9 \mathrm{e}$ & 5 a99 & $0 x^{2}$ \\
\hline & $0 \times 89$ & 0xfc2ed055 & 0xbcb66021 & 0x4ac97fd7 & 0xb42a129b & 0x60de7589 & $2 \mathrm{de}$ & 434 \\
\hline$\Delta^{b} V^{10}$ & \multicolumn{8}{|c|}{$\Delta^{b} v_{0}^{10}=(31), \Delta^{b} v_{5}^{10}=(16), \Delta^{b} v_{10}^{10}=(23), \Delta^{b} v_{15}^{10}=(23)$} \\
\hline \multirow{2}{*}{${ }_{a} V^{10}$} & $\mathrm{~d} 5$ & 621 & 0xc7139615 & & 0x4ded77e1 & 0x1ed1c43f & $0 \times 6$ & 72 \\
\hline & $3 b$ & ea2 & af & $9 c$ & $39 \mathrm{c}$ & $62 \mathrm{e}$ & 167 & $0 \mathrm{x}$ \\
\hline \multirow{2}{*}{${ }_{b} V^{10}$} & $\mathrm{~d} 5$ & $0 \times 7$ & $0 \mathrm{xc}$ & $0 \mathrm{x}^{2}$ & $7 \mathrm{e} 1$ & $43 \mathrm{f}$ & $\mathrm{dc}$ & $0 \mathrm{x}$ \\
\hline & 0x6e17623b & 0x4cd8bea 2 & Uxiec & $9 \mathrm{c}$ & $0 \mathrm{x} 53 \mathrm{~b} 6$ & 0x3972162e & 32167 & 0x4d9b2a4 \\
\hline \multirow{2}{*}{${ }_{c} V^{10}$} & $\mathrm{~d} 65$ & $0 \mathrm{x} 8 \mathrm{c}$ & $0 \times 537127 \mathrm{c} 9$ & $0 x a b c$ & $0 \mathrm{xc}$ & $f 49$ & $0 \times 66$ & $0 \times 8$ \\
\hline & $0 \mathrm{xccc}$ & 0x3d9aaba6 & $0 \times 84 \mathrm{ee} 030 \mathrm{c}$ & $0 x d$ & $0 \times 976348 \mathrm{e} 3$ & 0xfde7c240 & 0x1df99dc8 & 0x568a818c \\
\hline \multirow{2}{*}{${ }_{d} V^{10}$} & $0 x d a$ & $0 \mathrm{x} 8 \mathrm{~d} 12 \mathrm{db} 5$ & $0 \times 537127 \mathrm{c} 9$ & 0xabdb13a9 & 0xcaf27105 & 0x17ee5f49 & 0x66721638 & 0x8f333fbf \\
\hline & 0xccdc1196 & 0x3d9aaba6 & 0x846e030c & 0xda86539 & 0x976348e3 & 0xfde7c240 & 0x1df99dc8 & 0x560a818 \\
\hline
\end{tabular}


Table 13. Example for 6.5-round BLAKE2s with $y=28 \in \mathbb{X}_{256} \bigcap \mathbb{X}_{2 s}$.

\begin{tabular}{|c|c|c|c|c|c|c|c|c|}
\hline$\Delta M$ & \multicolumn{8}{|c|}{$\Delta^{b} m_{11}=(31), \Delta^{t} m_{5}=(28)$} \\
\hline \multirow{2}{*}{${ }_{a} M$} & 0xce9f1cc6 & 0x7f3a9b64 & 0x9e9ddc55 & 0x4553fa8c & 0xe2f4ad99 & $0 \times 33 a 0533 a$ & $0 \mathrm{x} 8 \mathrm{~b} 1 \mathrm{~d} 785 \mathrm{c}$ & 0xc7f56492 \\
\hline & 0xe5b2b205 & $0 x d 4$ & $0 \times 2 d$ & 0x18b03f68 & $0 \times 13$ & $9 \mathrm{f} 2$ & $0 \times 9$ & $\mathrm{ca04}$ \\
\hline \multirow{2}{*}{${ }_{b} M$} & & x7f3a9b64 & 0x9e9ddc55 & 0x4553fa8c & & 0x33a0533a & & 492 \\
\hline & $0 \mathrm{xe} 5$ & $0 x d 4$ & $0 \times 2$ & $3 f 68$ & $0 \times 1$ & $0 \mathrm{x} 1$ & 878 & $\mathrm{ca04}$ \\
\hline \multirow{2}{*}{${ }_{c} M$} & & b64 & & & & & & \\
\hline & 0 0xe5 & $0 x d 4$ & 00 & 668 & $0 \mathrm{x} 1$ & & & $\mathrm{a} 04$ \\
\hline \multirow{2}{*}{${ }_{d} M$} & 0xce9f1cc6 & $\times 7 f 3 a$ & $0 \mathrm{x} 9 \mathrm{e}$ & $\mathrm{fa} 8 \mathrm{c}$ & $0 x e 2$ & $0 \times 23$ & & 6492 \\
\hline & $0 x e 5$ & $0 x d 4$ & $0 \times 2 d$ & $0 \times 981$ & $0 \times 13$ & $0 \mathrm{x}$ & & $\mathrm{a} 04$ \\
\hline$\Delta^{t} V^{3.5}$ & \multicolumn{8}{|c|}{$\boldsymbol{\Delta}^{\mathbf{t}} \mathbf{v}_{\mathbf{1}}^{\mathbf{3 . 5}}=(\mathbf{2 9}), \Delta^{t} v_{11}^{3.5}=(28), \Delta^{t} v_{12}^{3.5}=(28,2)$} \\
\hline \multirow{2}{*}{${ }_{a} V^{3.5}$} & $\mathrm{a}$ & 0 & 84 & 0 & fe & 3 & 10 & $5 \mathrm{f}$ \\
\hline & $0 \times 3 c$ & $0 \times 6$ & $0 \times 2$ & b1 & $0 \mathrm{x}$ & $0 \mathrm{x} 4$ & 33 & $1 \mathrm{a} 0$ \\
\hline \multirow{2}{*}{${ }_{b} V^{3.5}$} & $36 \mathrm{~d}$ & $\mathrm{cfe}$ & 337 & $66 \mathrm{a}$ & b23 & $3 \mathrm{~b} 3$ & 985 & $00 \mathrm{a} 7$ \\
\hline & $0 \times 5$ & $0 \times 5 €$ & $0 \times 6$ & $5 \mathrm{ff}$ & $0 \mathrm{x}$ & $0 \times 3$ & 969 & $4 \mathrm{da}$ \\
\hline \multirow{2}{*}{${ }_{c} V^{3.5}$} & $c 4 a$ & $0 \times 6$ & & a9 & $2 \mathrm{fe}$ & Da3 & $\mathrm{d} 0$ & $55 \mathrm{f}$ \\
\hline & $0 \mathrm{x} 3 \mathrm{cc}$ & $0 \times 6 c$ & $0 \times 2 b$ & $0 \times 1$ & $0 \times 9$ & $0 \mathrm{x} 4$ & 233 & $1 \mathrm{a} 0$ \\
\hline \multirow{2}{*}{${ }_{d} V^{3.5}$} & $86 \mathrm{~d}$ & $0 \times 3$ & $0 \times 7$ & $6 a$ & 23 & $3 \mathrm{~b} 3$ & 85 & $0 \mathrm{a} 7$ \\
\hline & $0 \times 5$ & 0x5edbf5ae & $\mathrm{b} 25$ & $5 \mathrm{ff}$ & 836 & 100 & 969 & $4 \mathrm{da}$ \\
\hline$\Delta^{b} V^{10}$ & \multicolumn{8}{|c|}{$\Delta^{b} v_{0}^{10}=(31), \Delta^{b} v_{5}^{10}=(16), \Delta^{b} v_{10}^{10}=(23), \Delta^{b} v_{15}^{10}=(23)$} \\
\hline \multirow{2}{*}{${ }_{a} V^{10}$} & $0 \times 94$ & $0 x 422107 a b$ & 0x3a682330 & 0x2f8bd4f1 & 0xeead389 & $0 \times 21 \mathrm{e} 907 \mathrm{ec}$ & $0 \times 17$ & 62 \\
\hline & 0x229a3e13 & $\mathrm{c} 2 \mathrm{c}$ & $7 \mathrm{~d} 4 \mathrm{a}$ & 0xf1 & $0 \times 5 d$ & 0x1be & $0 \times 78$ & $0 \mathrm{x} \varepsilon$ \\
\hline \multirow{2}{*}{${ }_{b} V^{10}$} & $0 \times 145 \mathrm{cf} 52 \mathrm{e}$ & $0 x 422107 a b$ & 0x3a682330 & 0x2f8bd4f1 & 0xeead389 & 0x21e807ec & 0x17138a07 & 0xae021462 \\
\hline & 0x229a3e13 & $0 \times 3 c 623 c 2 c$ & $0 \times 64 \mathrm{~b} 27 \mathrm{~d} 4 \mathrm{a}$ & 0xf1d0e09a & 0x5df5abad & 0x1be8464a & 0x7890983a & $0 \times 85 a 88868$ \\
\hline \multirow{2}{*}{${ }_{c} V^{10}$} & 0xc136da56 & 0xe91ba476 & 0xfa9ad265 & 0x6b4d2f9e & 0x68ef06c8 & 0x9ab4757a & 0xe63456e0 & $0 \mathrm{x} 8818 \mathrm{e} 9 \mathrm{~d} 4$ \\
\hline & 0x5da1784c & $0 \times 57$ ecd $14 b$ & 0xcb0788b8 & 0xf3148edf & 0xa19d7f24 & 0xf17b5303 & 0x9ec70b70 & 0x2f763872 \\
\hline \multirow{2}{*}{${ }_{d} V^{10}$} & 0x4136da56 & 0xe91ba476 & 0xfa9ad265 & 0x6b4d2f9e & $0 \times 68$ ef06c8 & 0x9ab5757a & 0xe63456e0 & $0 \times 8818 \mathrm{e} 9 \mathrm{~d} 4$ \\
\hline & 0x5da1784c & $0 \times 57$ ecd $14 b$ & 0xcb8788b8 & 0xf3148edf & 0xa19d7f24 & 0xf17b5303 & 0x9ec70b70 & 0x2ff63872 \\
\hline
\end{tabular}


Table 14. Example for 6.5-round BLAKE-512 with $y=9 \in \mathbb{X}_{512} \bigcap \mathbb{X}_{2 b}$.

\begin{tabular}{|c|c|c|c|c|}
\hline$\Delta M$ & \multicolumn{4}{|c|}{$\Delta^{b} m_{11}=(63), \Delta^{t} m_{5}=(9)$} \\
\hline \multirow{4}{*}{${ }_{a} M$} & 0x9c1860c444a6aff4 & 0xc95a712fd5a29b72 & 0x6e5c6811448b300f & 0x5c0af45531e396d3 \\
\hline & 0x679dee5280c15ad0 & 0x329f5347ccb9bf64 & 0x297828d3ec89e9d0 & 0xa55ffc029ea78609 \\
\hline & 0xef01f63ec485f87d & 0x86560936e36d9dff & 0xfd9674bb724d62e0 & 0x9c03f6f64a96659f \\
\hline & 0xe3666bd816053d27 & 0xe4669665a4a0a440 & 0x1cbf0c93a121eb09 & 0x65a6a90ac809c019 \\
\hline \multirow{4}{*}{${ }_{b} M$} & 0x9c1860c444a6a9f4 & 0xc95a712fd5a29b72 & 0x6e5c6811448b300f & $0 \times 5 c 0 a f 45531 \mathrm{e} 396 \mathrm{~d} 3$ \\
\hline & 0x679dee5280c15ad0 & 0x329f5347ccb9bf64 & 0x297828d3ec89e9d0 & 0xa55ffc029ea78609 \\
\hline & 0xef01f63ec485f87d & 0x86560936e36d9dff & 0xfd9674bb724d62e0 & 0x1c03f6f64a96659f \\
\hline & 0xe3666bd816053d27 & 0xe4669665a4a0a440 & 0x1cbf0c93a121eb09 & 0x65a6a90ac809c019 \\
\hline \multirow{4}{*}{${ }_{c} M$} & 0x9c1860c444a6a9f4 & 0xc95a712fd5a29b72 & 0x6e5c6811448b300f & 0x5c0af45531e396d3 \\
\hline & 0x679dee5280c15ad0 & 0x329f5347ccb9bd64 & 0x297828d3ec89e9d0 & 0xa55ffc029ea78609 \\
\hline & 0xef01f63ec485f87d & 0x86560936e36d9dff & 0xfd9674bb724d62e0 & 0x9c03f6f64a96659f \\
\hline & 0xe3666bd816053d27 & 0xe4669665a4a0a440 & 0x1cbf0c93a121eb09 & 0x65a6a90ac809c019 \\
\hline \multirow{4}{*}{${ }_{d} M$} & 0x9c1860c444a6a9f4 & 0xc95a712fd5a29b72 & 0x6e5c6811448b300f & 0x5c0af45531e396d3 \\
\hline & 0x679dee5280c15ad0 & 0x329f5347ccb9bd64 & 0x297828d3ec89e9d0 & 0xa55ffc029ea78609 \\
\hline & 0xef01f63ec485f87d & 0x86560936e36d9dff & 0xfd9674bb724d62e0 & 0x1c03f6f64a96659f \\
\hline & 0xe3666bd816053d27 & 0xe4669665a4a0a440 & 0x1cbf0c93a121eb09 & 0x65a6a90ac809c019 \\
\hline$\Delta^{t} V^{3.5}$ & \multicolumn{4}{|c|}{$\boldsymbol{\Delta}^{\mathrm{t}} \mathbf{v}_{1}^{\mathbf{3 . 5}}=\boldsymbol{\phi}, \Delta^{t} v_{11}^{3.5}=(9), \Delta^{t} v_{12}^{3.5}=(41,9)$} \\
\hline \multirow{4}{*}{${ }_{a} V^{3.5}$} & 0xc87af7255a6ec986 & 0xc59be5b07a4418d7 & 0x5295eb179fee042c & 0x4f87d569d171c685 \\
\hline & 0xc1c24f85f094b263 & 0xbc711b20878eb4ea & 0x1cda016fcf08ee93 & 0x878f439bd1398fec \\
\hline & 0x982d7a384b8549bb & 0x29cd6958f1a234c3 & 0xb81579ed9e3eff45 & 0xbfba600ee495e360 \\
\hline & 0x4d5e10f24eba6506 & 0x4f8a20a0c7164ef8 & 0x4156d917e0e33e7b & 0x8f204cb6dc806747 \\
\hline \multirow{4}{*}{${ }_{b} V^{3.5}$} & $0 \times 57926274 \mathrm{c} 228 \mathrm{f} 656$ & 0x5ac46fa843cda867 & 0x936f1f621381dad4 & 0xbd0f73ec836d47bc \\
\hline & 0xbac8918094537e74 & 0x1edec058ea817875 & 0xc5bf41aeadf39382 & 0x4149082191041e60 \\
\hline & 0x9fd575b7fe10ace3 & 0x8fed3642acc17d51 & 0x1ded33ae6ee468ba & 0x5365299759c0a42 \\
\hline & 0x89f06ef09e1612ee & 0xe597ede91683a2d8 & 0x389825cb39587e4f & 0xff48c413164455c3 \\
\hline \multirow{4}{*}{${ }_{c} V^{3.5}$} & 0xc87af7255a6ec986 & 0xc59be5b07a4418d7 & 0x5295eb179fee042c & 0x4f87d569d171c685 \\
\hline & 0xc1c24f85f094b263 & 0xbc711b20878eb4ea & 0x1cda016fcf08ee93 & 0x878f439bd1398fec \\
\hline & 0x982d7a384b8549bb & 0x29cd6958f1a234c3 & 0xb81579ed9e3eff45 & 0xbfba600ee495e160 \\
\hline & 0x4d5e12f24eba6706 & $0 \mathrm{x} 4 \mathrm{f} 8 \mathrm{a} 20 \mathrm{a} 0 \mathrm{c} 7164 \mathrm{ef} 8$ & 0x4156d917e0e33e7b & 0x8f204cb6dc806747 \\
\hline \multirow{4}{*}{${ }_{d} V^{3.5}$} & $0 \mathrm{x} 57926274 \mathrm{c} 228 \mathrm{f} 656$ & $0 \times 5 a c 46 f a 843 c d a 867$ & 0x936f1f621381dad4 & 0xbd0f73ec836d47bc \\
\hline & 0xbac8918094537e74 & 0x1edec058ea817875 & 0xc5bf41aeadf39382 & 0x4149082191041e60 \\
\hline & 0x9fd575b7fe10ace3 & 0x8fed3642acc17d51 & 0x1ded33ae6ee468ba & $0 \times 5365299759 \mathrm{c} 0842$ \\
\hline & 0x89f06cf09e1610ee & 0xe597ede91683a2d8 & 0x389825cb39587e4f & 0xff48c413164455c3 \\
\hline$\Delta^{b} V^{10}$ & \multicolumn{4}{|c|}{$\Delta^{b} v_{0}^{10}=(63), \Delta^{b} v_{5}^{10}=(36), \Delta^{b} v_{10}^{10}=(47), \Delta^{b} v_{15}^{10}=(47)$} \\
\hline \multirow{4}{*}{${ }_{a} V^{10}$} & 0x1b404ab31fbe9343 & 0xc01ae4355f49855f & 0xf52deb99e6d25dee & 0xba1e74d813d9e09c \\
\hline & 0x1d4142ceee078181 & 0x8c7261a65899559 & 0x780312586191c134 & 0x86c7c29f8161a9ac \\
\hline & 0x77f4ec97a373e3dd & 0x7068ac849086f0c3 & 0xfc3c0163cdc3f7b9 & 0x52d68b2940599cfa \\
\hline & 0x59ad1c82831be8f7 & 0x74d99e11568eb396 & 0x3552275c6ddcf7a3 & 0x8dfe0979b5e83dbd \\
\hline \multirow{4}{*}{${ }_{b} V^{10}$} & 0x9b404ab31fbe9343 & 0xc01ae4355f49855f & 0xf52deb99e6d25dee & 0xba1e74d813d9e09c \\
\hline & 0x1d4142ceee078181 & 0x8c7260a65899559 & 0x780312586191c134 & 0x86c7c29f8161a9ac \\
\hline & 0x77f4ec97a373e3dd & 0x7068ac849086f0c3 & 0xfc3c8163cdc3f7b9 & 0x52d68b2940599cfa \\
\hline & 0x59ad1c82831be8f7 & 0x74d99e11568eb396 & 0x3552275c6ddcf7a3 & 0x8dfe8979b5e83dbd \\
\hline \multirow{4}{*}{${ }_{c} V^{10}$} & 0xcc0a78ca6c133737 & 0xa6a12a75a2ab0a78 & 0xaaff3e032bf0964f & 0x6a833f52c06326f8 \\
\hline & 0x1571fbe8468d6869 & 0x224b394014f172d8 & 0x72a0866c8eb1dfcc & 0x4af2b98060eea9bb \\
\hline & 0xe7f5b1b201006785 & 0xa57c9190f805d201 & 0xdea0ecffe0219e24 & 0xbbec25c771762bfb \\
\hline & 0xd312a8ab8e4df740 & 0xd9a366032739ede2 & 0xb8d5bfa962e8d684 & 0xb122b4542c543d9d \\
\hline \multirow{4}{*}{${ }_{d} V^{10}$} & 0x4c0a78ca6c133737 & 0xa6a12a75a2ab0a78 & 0xaaff3e032bf0964f & 0x6a833f52c06326f8 \\
\hline & 0x1571fbe8468d6869 & 0x224b395014f172d8 & 0x72a0866c8eb1dfcc & 0x4af2b98060eea9bb \\
\hline & 0xe7f5b1b201006785 & 0xa57c9190f805d201 & 0xdea06cffe0219e24 & 0xbbec25c771762bfb \\
\hline & 0xd312a8ab8e4df740 & 0xd9a366032739ede2 & 0xb8d5bfa962e8d684 & 0xb12234542c543d9d \\
\hline
\end{tabular}


Table 15. Example for 6.5-round BLAKE2b with $y=9 \in \mathbb{X}_{512} \bigcap \mathbb{X}_{2 b}$.

\begin{tabular}{|c|c|c|c|c|}
\hline$\Delta M$ & \multicolumn{4}{|c|}{$\Delta^{b} m_{11}=(63), \Delta^{t} m_{5}=(9)$} \\
\hline \multirow{4}{*}{${ }_{a} M$} & 0x3cec6965bf357a5 & 0x3efa6687e114e70d & 0x6fe9d72277e832e4 & 0x60574e830fad0b27 \\
\hline & 0x1bad3b4b1257079e & 0x43b8e8ebf1bc4557 & 0xc553a639b52984b0 & 0x95bd9c03c94695e5 \\
\hline & 0xc4e9f58d840c74c9 & 0x2186128d765d51b0 & 0x10bc4fee175e6c82 & 0x18ddcb4d4ac938ee \\
\hline & 0x5cf2d8b6cf1ea3ce & 0x3ec5aa659dacedf5 & 0xadf91c482e6b4506 & 0xa34876 \\
\hline \multirow{4}{*}{${ }_{b} M$} & 0x3cec6965bf357a5 & 0x3efa6687e114e70d & 0x6fe9d72277e832e4 & 0x60574e830fad0b27 \\
\hline & 0x1bad3b4b1257079e & 0x43b8e8ebf1bc4557 & 0xc553a639b52984b0 & 0x95bd9c03c94695e5 \\
\hline & 0xc4e9f58d840c74c9 & 0x2186128d765d51b0 & 0x10bc4fee175e6c82 & 0x98ddcb4d4ac938ee \\
\hline & 0x5cf2d8b6cf1ea3ce & 0x3ec5aa659dacedf5 & 0xadf91c482e6b4506 & 0xa34876d149007c7b \\
\hline \multirow{4}{*}{${ }_{c} M$} & 0x3cec6965bf357a5 & 0x3efa6687e114e70d & 0x6fe9d72277e832e4 & 0x60574 \\
\hline & 0x1bad3b4b1257079e & 0x43b8e8ebf1bc4757 & 0xc553a639b52984b0 & 0x95bd9c03c94695e5 \\
\hline & 0xc4e9f58d840c74c9 & 0x2186128d765d51b0 & 0x10bc4fee175e6c82 & 0x18ddcb4d4ac938ee \\
\hline & 0x5cf2d8b6cf1ea3ce & 0x3ec5aa659dacedf5 & 0xadf91c482e6b4506 & 0xa34876d149007c7b \\
\hline \multirow{4}{*}{${ }_{d} M$} & 0x3cec6965bf357a5 & 0x3efa6687e114e70d & 0x6fe9d72277e832e4 & 0x60574e830fad0b27 \\
\hline & 0x1bad3b4b1257079e & 0x43b8e8ebf1bc4757 & 0xc553a639b52984b0 & 0x95bd9 \\
\hline & 0xc4e9f58d840c74c9 & 0x2186128d765d51b0 & 0x10bc4fee175e6c82 & 0x98ddc \\
\hline & 0x5cf2d8b6cf1ea3ce & 0x3ec5aa659dacedf5 & 0xadf91c482e6b4506 & 0xa34876d149007c7b \\
\hline$V^{3.5}$ & \multicolumn{4}{|c|}{$\boldsymbol{\Delta}^{\mathbf{t}} \mathbf{v}_{\mathbf{1}}^{\mathbf{3 . 5}}=(\mathbf{1 0}), \Delta^{t} v_{11}^{3.5}=(9), \Delta^{t} v_{12}^{3.5}=(41,9)$} \\
\hline \multirow{4}{*}{${ }_{a} V^{3.5}$} & 0xa8f431bca7166664 & 0x2bce $47208 \mathrm{c} 2 \mathrm{~b} 479 \mathrm{~d}$ & 0x2554f082eb89d530 & 0x12b06bc7f71ebe12 \\
\hline & $0 \mathrm{x} 5 \mathrm{~d} 733 \mathrm{~d} 5 \mathrm{fa} 41457 \mathrm{fc}$ & 0xae2b3d68d8adfe2f & 0xe03c7fa88285b93d & 0xe134f22af656a9d9 \\
\hline & 0x8bcd47a74a5e35a2 & 0x21098bd0acfbc078 & 0x9d0ddd6c2403d2ab & 0xf0dbb0c6a9a392c5 \\
\hline & 0xd72aa227f3c2a651 & 0x406e07f8eec1929f & 0x863da54a0653fe1f & 0xefb750af7de2c392 \\
\hline \multirow{4}{*}{${ }_{b} V^{3.5}$} & 0x63b1930b9a252aff & 0xd754470ae2a5de96 & 0x1b39d8f987ec3762 & 0x201afad51a642cb1 \\
\hline & $0 \mathrm{x} 1 \mathrm{~d} 5 \mathrm{c} 8 \mathrm{e} 5 \mathrm{fb} 50 \mathrm{c} 1 \mathrm{c} 68$ & 0x709103f9ba538f43 & 0xb847dad7a1bf8a56 & 0xa59f9b63902edb4 \\
\hline & 0x40d96db5d9d3b546 & 0x332aed26d86aceaa & 0x424eaab611c9c6f & $0 \times 802 \mathrm{~b} 6 \varepsilon$ \\
\hline & $0 x 110 \mathrm{~cd} 82 \mathrm{fdac} 384 \mathrm{dd}$ & 0xa93fe8a10201b57b & 0x49eed3d94b17685a & 0xcdf2a00fd5300651 \\
\hline \multirow{4}{*}{${ }_{c} V^{3.5}$} & 0xa8f431bca7166664 & 0x2bce $47208 \mathrm{c} 2 \mathrm{~b} 439 \mathrm{~d}$ & 0x2554f082eb89d530 & 0x12b06bc7f71ebe12 \\
\hline & $0 \times 5 d 733 d 5 f a 41457 f c$ & 0xae2b3d68d8adfe2f & 0xe03c7fa88285b93d & 0xe134f22af656a9d9 \\
\hline & 0x8bcd47a74a5e35a2 & 0x21098bd0acfbc078 & 0x9d0ddd6c2403d2ab & 0xf0dbb0c6a9a390c5 \\
\hline & 0xd72aa027f3c2a451 & 0x406e07f8eec1929f & 0x863da54a0653fe1f & 0xefb750af7de2c392 \\
\hline \multirow{4}{*}{${ }_{d} V^{3.5}$} & 0x63b1930b9a252aff & 0xd754470ae2a5da96 & 0x1b39d8f987ec3762 & $51 \mathrm{a} 642 \mathrm{cb} 1$ \\
\hline & 0x1d5c8e5fb50c1c68 & 0x709103f9ba538f43 & 0xb847dad7a1bf8a56 & 0xa59f9b63902edb4 \\
\hline & 0x40d96db5d9d3b546 & 0x332aed26d86aceaa & 0x424eaab611c9c6f & 0x802b683db9ac56b9 \\
\hline & 0x110cda2fdac386dd & 0xa93fe8a10201b57b & 0x49eed3d94b17685a & fd5300651 \\
\hline$\Delta^{b} V^{10}$ & $\Delta^{b} v_{0}^{1}$ & $=(63), \Delta^{b} v_{5}^{10}=(48)$, & $\Delta^{b} v_{10}^{10}=(47), \Delta^{b} v_{15}^{10}$ & $=(47)$ \\
\hline \multirow{4}{*}{${ }_{a} V^{10}$} & 0x96ace3d164600933 & 0x6785c14493444a3d & 0xadc3b5f6dbc8c992 & 0xada06 \\
\hline & 0xcb06b797a6152dbe & 0xf701f3e0f76be4cb & 0xf4baf3238d75bdb6 & 0xb71965677688de57 \\
\hline & 0xaa494db2c0d12db8 & 0x10ab8d9652485fcf & 0xb97f5a3ef869239f & 0x560aff2ec6a0d95f \\
\hline & 0x1597013f79b484d1 & 0x182beacffdc6ec05 & 0x6802644a544f6271 & 0x59ac761a17acecca \\
\hline \multirow{4}{*}{${ }_{b} V^{10}$} & 0x16ace3d164600933 & 0x6785c14493444a3d & 0xadc3b5f6dbc8c992 & $115 \mathrm{f} 42653 \mathrm{a}$ \\
\hline & 0xcb06b797a6152dbe & 0xf700f3e0f76be4cb & 0xf4baf3238d75bdb6 & 0xb71965677688de57 \\
\hline & 0xaa494db2c0d12db8 & 0x10ab8d9652485fcf & 0xb97fda3ef869239f & 0x560aff2ec6a0d95f \\
\hline & 0x1597013f79b484d1 & 0x182beacffdc6ec05 & $0 \times 6802644 a 544 f 6271$ & 0x59acf61a17acecca \\
\hline \multirow{4}{*}{${ }_{c} V^{10}$} & 0xe8d4f6a3aa68e9d6 & 0x1ba5272a94ed608d & 0x51b3a429d5ee6873 & 0x50af4c1bb7b31dd 2 \\
\hline & 0x738835de6bff309d & 0xc5fc88e668afef14 & 0x1671fea856c55b2d & 0xd04b446c31b59a1b \\
\hline & 0x8f120d94bae51fa1 & 0x5be58c40a2d2c0a9 & 0xe9c1de5ac5992a67 & 0xa307fd45e31b7817 \\
\hline & 0xbd4864acd0f2e4bc & 0x4a8a43605d94a9b4 & 0x16e63ec7c12bc056 & 0x30e48769ae169de0 \\
\hline \multirow{4}{*}{${ }_{d} V^{10}$} & 0x68d4f6a3aa68e9d6 & 0x1ba5272a94ed608d & 0x51b3a429d5ee6873 & 0x50af4c1bb7b31dd2 \\
\hline & 0x738835de6bff309d & 0xc5fd88e668afef14 & 0x1671fea856c55b2d & 0xd04b446c31b59a1b \\
\hline & 0x8f120d94bae51fa1 & 0x5be58c40a2d2c0a9 & 0xe9c15e5ac5992a67 & 0xa307fd45e31b7817 \\
\hline & 0xbd4864acd0f2e4bc & 0x4a8a43605d94a9b4 & 0x16e63ec7c12bc056 & 0x30e40769ae169de0 \\
\hline
\end{tabular}

